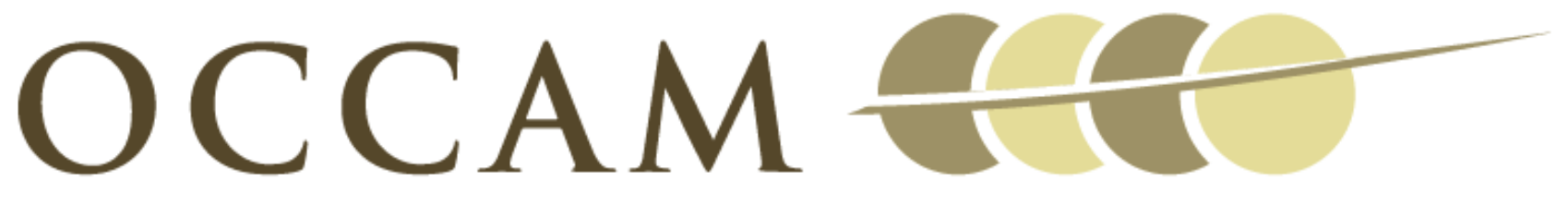

OXFORD CENTRE FOR COLLABORATIVE APPLIED MATHEMATICS

\author{
Report Number 11/51
}

A non-local vector calculus,non-local volume-constrained problems, and non-local balance laws

by

Jqiang Du, Max Gunzburger, R. B. Lehoucq, and Kun Zhou

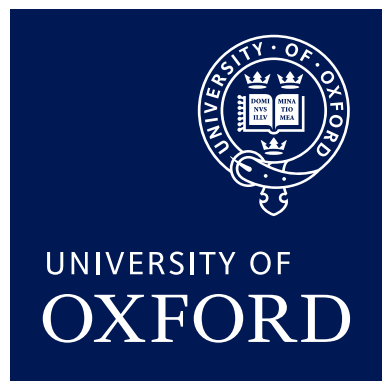

Oxford Centre for Collaborative Applied Mathematics Mathematical Institute 24 - 29 St Giles'

Oxford

OX1 3LB

England 



\title{
A NONLOCAL VECTOR CALCULUS, NONLOCAL VOLUME-CONSTRAINED PROBLEMS, AND NONLOCAL BALANCE LAWS
}

\author{
QIANG DU \\ Department of Mathematics, Pennsylvania State University \\ University Park, PA 16802 \\ qdu@math.psu.edu \\ MAX GUNZBURGER \\ Department of Scientific Computing, Florida State University \\ Tallahassee FL 32305-4120 \\ gunzburg@fsu.edu \\ R. B. LEHOUCQ \\ Sandia National Laboratories \\ P.O. Box 5800, MS 1320, Albuquerque, NM 87185-1320 \\ rblehou@sandia.gov \\ KUN ZHOU \\ Department of Mathematics, Pennsylvania State University \\ University Park, PA 16802 \\ zhou@math.psu.edu \\ Received (Day Month Year) \\ Revised (Day Month Year) \\ Communicated by (xxxxxxxxxx)
}

\begin{abstract}
A vector calculus for nonlocal operators is developed, including the definition of nonlocal divergence, gradient, and curl operators and the derivation of the corresponding adjoints operators. Nonlocal analogs of several theorems and identities of the vector calculus for differential operators are also presented. Relationships between the nonlocal operators and their differential counterparts are established, first in a distributional sense and then in a weak sense by considering weighted integrals of the nonlocal adjoint operators. The nonlocal calculus gives rise to volume-constrained problems that are analogous to elliptic boundary-value problems for differential operators; this is demonstrated via some examples. Another application is posing abstract nonlocal balance laws and deriving the corresponding nonlocal field equations.
\end{abstract}

Keywords: nonlocal operators, vector calculus, volume-constrained problems, balance laws, peridynamics, nonlocal diffusion

AMS Subject Classification: 26B12, 26B15, 26B20, 45A05, 45P05, 45E99, 46F12 


\section{Introduction}

We introduce a vector calculus for nonlocal operators that mimics the classical vector calculus for differential operators. We define nonlocal analogs of the divergence, gradient, and curl operators and deduce the corresponding nonlocal adjoint operators. Nonlocal analogs of the Gauss theorem and the Green's identities of the vector calculus for differential operators are also derived. We establish relationships between the nonlocal operators and their differential counterparts. The nonlocal vector calculus can be used to define nonlocal volume-constrained problems that are analogous to boundary-value problems for partial differential operators. In addition, The nonlocal vector calculus we develop has an important application to balance laws ${ }^{\mathrm{a}}$ that are nonlocal in the sense that subregions not in direct contact may have a non-vanishing interaction. This is accomplished by defining a nonlocal flux in terms of interactions between disjoint regions of positive measure possibly sharing no common boundary. Our nonlocal vector calculus, then, provides an alternative to standard approaches for circumventing the technicalities associated with the lack of sufficient regularity in local balance laws.

Preliminary attempts at a nonlocal calculus are found in Refs. 8 and 9 which included applications to image processing and steady-state diffusion, respectively. In particular, the authors of Ref. 8 cite Ref. 22 where a discrete nonlocal divergence and gradient are introduced within the context of machine learning; see also of of Refs. 4, 11, and 13 where a discrete calculus is also discussed. However, the discussion in these papers is limited to scalar problems. In contrast, this paper extends the ideas in Refs. 8 and 9 to vector and tensor fields and beyond the consideration of image processing and steady-state diffusion. For example, the ideas presented here enable an abstract formulation of the balance laws of momentum and energy and for the peridynamic theory for solid mechanics ${ }^{\mathrm{b}}$ that parallels the classical vector calculus formulation of the balance laws of elasticity. The nonlocal vector calculus presented in this paper, however, is sufficiently general that we envisage application to balance laws beyond those of elasticity, e.g., to the laws of fluid mechanics and electromagnetics.

The paper is organized as follows. The remainder of this section is devoted to establishing notation. In Section 2, the notions of local and nonlocal fluxes into or out off a region are briefly compared and contrasted. In Section 3, the nonlocal divergence, gradient, and curl operators are introduced as are the corresponding adjoint operators, several vector identities, and other results about the operators. The nonlocal vector calculus is developed in Section 4; in particular, nonlocal inte-

\footnotetext{
${ }^{a} \mathrm{~A}$ balance law postulates that the rate of change of an extensive quantity over any subregion of a body is given by the rate at which that quantity is produced in the subregion minus the flux out of the subregion through its boundary.

${ }^{\mathrm{b}}$ Peridynamics was introduced in Refs. 17 and 19; Ref. 20 reviews the peridynamic balance laws of momentum and energy and provides many citations for the peridynamic theory and its applications. See Section 7.2 for a brief discussion.
} 
gral theorems and nonlocal Green's identities are derived. In Section 5, connections between the nonlocal operators and distributional and weak representations of the associated classical differential operators are made. The connections made in Section 5 justify the use of the terminology "nonlocal divergence, gradient, and curl" to refer to the nonlocal operators defined in Section 3. Sections 6 and 7 deal with applications of the nonlocal vector calculus. In Section 6, examples are given of nonlocal volume-constrained problems formulated in terms of the nonlocal operators. Then, in Section 7, a brief review of the conventional notion of a balance law is provided after which abstract nonlocal balance laws are discussed. The notion of nonlocal fluxes discussed in Section 2 is used in developing nonlocal balance laws and the vector calculus developed in Section 4 plays a crucial role in transforming balance laws into field equations. Also, in Section 7, a brief discussion is given of the application of our nonlocal vector calculus to the peridynamic theory for continuum mechanics.

Throughout, wherever it is illuminating, we associate definitions and results of the nonlocal vector calculus with the analogous definitions and results of the classical differential vector calculus.

\subsection{Notation}

We have need of two types of functions and two types of nonlocal operators. Point functions refer to functions defined at points whereas two-point functions refer to functions defined for pairs of points. Point operators map two-point functions to point functions whereas two-point operators map point functions to two-point functions so that the nomenclature for operators refer to their ranges. Point and twopoint operators are both nonlocal. Point operators involve integrals of two-point functions whereas two-point operators explicitly involve point functions evaluated at two different points.

We now make more precise the definitions given above. Points in $\mathbb{R}^{n}$ are denoted by the vectors $\mathbf{x}, \mathbf{y}$, or $\mathbf{z}$ and the natural Cartesian basis is denoted by $\mathbf{e}_{1}, \ldots, \mathbf{e}_{n}$. Let $m, k$, and $n$ denote positive integers. For $\Omega \subseteq \mathbb{R}^{n}$, functions from $\Omega$ into $\mathbb{R}^{m \times k}$ or $\mathbb{R}^{m}$ or $\mathbb{R}$ are referred to as point functions or point mappings and are denoted by Roman letters, upper-case bold for tensors, lower-case bold for vectors, and plain face for scalars, respectively, e.g., $\mathbf{U}(\mathbf{x}), \mathbf{u}(\mathbf{x})$, and $u(\mathbf{x})$, respectively. Functions from $\Omega \times \Omega$ into $\mathbb{R}^{m \times k}$ or $\mathbb{R}^{m}$ or $\mathbb{R}$ are referred to as two-point functions or two-point mappings and are denoted by Greek letters, upper-case bold for tensors, lowercase bold for vectors, and plain face for scalars, respectively, e.g., $\mathbf{\Psi}(\mathbf{x}, \mathbf{y}), \boldsymbol{\psi}(\mathbf{x}, \mathbf{y})$, and $\psi(\mathbf{x}, \mathbf{y})$, respectively. Symmetric and antisymmetric scalar two-point functions $\psi(\mathbf{x}, \mathbf{y})$ satisfy $\psi(\mathbf{x}, \mathbf{y})=\psi(\mathbf{y}, \mathbf{x})$ and $\psi(\mathbf{x}, \mathbf{y})=-\psi(\mathbf{y}, \mathbf{x})$, respectively, and similarly for vector and tensor two-point functions. A useful observation is that

$$
\text { if } \psi(\mathbf{x}, \mathbf{y}) \text { is antisymmetric, then } \int_{\Omega} \int_{\Omega} \psi(\mathbf{x}, \mathbf{y}) d \mathbf{y} d \mathbf{x}=0 \quad \forall \Omega \subseteq \mathbb{R}^{n}
$$

and similarly for antisymmetric vector and tensor two-point functions. 
For the sake of notational simplicity, in much of the rest of the paper, we introduce the following notation:

$$
\begin{aligned}
\boldsymbol{\alpha}:=\boldsymbol{\alpha}(\mathbf{x}, \mathbf{y}) & \boldsymbol{\alpha}^{\prime}:=\boldsymbol{\alpha}(\mathbf{y}, \mathbf{x}) & \psi:=\psi(\mathbf{x}, \mathbf{y}) & \psi^{\prime}:=\psi(\mathbf{y}, \mathbf{x}) \\
\mathbf{u}:=\mathbf{u}(\mathbf{x}) & \mathbf{u}^{\prime}:=\mathbf{u}(\mathbf{y}) & u:=u(\mathbf{x}) & u^{\prime}:=u(\mathbf{y})
\end{aligned}
$$

and similarly for other functions.

The $\operatorname{dot}$ (or inner) product of two vectors $\mathbf{u}, \mathbf{v} \in \mathbb{R}^{m}$ is denoted by $\mathbf{u} \cdot \mathbf{v} \in \mathbb{R}$; the dyad (or outer) product is denoted by $\mathbf{u} \otimes \mathbf{w} \in \mathbb{R}^{m \times k}$ whenever $\mathbf{w} \in \mathbb{R}^{k}$; given a second-order tensor (matrix) $\mathbf{U} \in \mathbb{R}^{k \times m}$, the tensor-vector (or matrix-vector) product is denoted by $\mathbf{U} \cdot \mathbf{v}$ and is given by the vector whose components are the dot products of the corresponding rows of $\mathbf{U}$ with $\mathbf{v} .{ }^{\mathrm{c}}$ For $n=3$, the cross product of two vectors $\mathbf{u}$ and $\mathbf{v}$ is denoted by $\mathbf{u} \times \mathbf{v} \in \mathbb{R}^{3}$. The Frobenius product of two second-order tensors $\mathbf{A} \in \mathbb{R}^{m \times k}$ and $\mathbf{B} \in \mathbb{R}^{m \times k}$, denoted by $\mathbf{A}: \mathbf{B}$, is given by the sum of the element-wise product of the two tensors. The trace of $\mathbf{B} \in \mathbb{R}^{m \times m}$, denoted by $\operatorname{tr}(\mathbf{B})$, is given by the sum of the diagonal elements of $\mathbf{B}$.

Inner products in $L^{2}(\Omega)$ and $L^{2}(\Omega \times \Omega)$ are defined in the usual manner. For example, for vector functions, we have

$$
\begin{cases}(\mathbf{u}, \mathbf{v})_{\Omega}=\int_{\Omega} \mathbf{u} \cdot \mathbf{v} d \mathbf{x} & \text { for } \mathbf{u}(\mathbf{x}), \mathbf{v}(\mathbf{x}) \in \Omega \\ (\boldsymbol{\mu}, \boldsymbol{\nu})_{\Omega \times \Omega}=\int_{\Omega} \int_{\Omega} \boldsymbol{\mu} \cdot \boldsymbol{\nu} d \mathbf{y} d \mathbf{x} & \text { for } \boldsymbol{\mu}(\mathbf{x}, \mathbf{y}), \boldsymbol{\nu}(\mathbf{x}, \mathbf{y}) \in \Omega\end{cases}
$$

with analogous expressions involving the Frobenius product and the ordinary product for tensor and scalar functions, respectively.

\section{Nonlocal fluxes and nonlocal action-reaction principles}

A key concept in the development of a vector calculus is the notion of a flux which accounts for the interaction of points in a domain with points outside the domain. As a result, the notion of a flux is also fundamental to the understanding of balance laws in mechanics, heat transfer, and many other settings; see Section 7. In the classical setting of local interactions, that interaction occurs at the boundary of the domain, whereas in the nonlocal case, the interaction must occur over volumes external to the domain. In order to contrast the notion of a nonlocal flux with the classical local flux, we begin by briefly reviewing the latter notion.

\subsection{Local fluxes}

Let $\Omega_{1} \subset \mathbb{R}^{n}$ and $\Omega_{2} \subset \mathbb{R}^{n}$ denote two disjoint open regions. If $\Omega_{1}$ and $\Omega_{2}$ have a nonempty common boundary $\partial \Omega_{12}:=\bar{\Omega}_{1} \cap \bar{\Omega}_{2}$, then, for a vector-valued function

${ }^{\mathrm{c}}$ In matrix notation, the inner, outer, matrix-vector products are given by $\mathbf{x} \cdot \mathbf{y}=\mathbf{x}^{T} \mathbf{y}, \mathbf{x} \otimes \mathbf{y}=\mathbf{x y} \mathbf{y}^{T}$, and $\mathbf{U} \cdot \mathbf{v}=\mathbf{U} \mathbf{v}$. 
$\mathbf{q}(\mathbf{x})$, the expression

$$
\int_{\partial \Omega_{12}} \mathbf{q} \cdot \mathbf{n}_{1} d A
$$

represents the classical local flux out of $\Omega_{1}$ into $\Omega_{2}$, where $\mathbf{n}_{1}$ denotes the unit normal on $\partial \Omega_{12}$ pointing outward from $\Omega_{1}$ and $d A$ denotes a surface measure in $\mathbb{R}^{n} ; \mathbf{q} \cdot \mathbf{n}_{1}$ is referred as the flux density along $\partial \Omega_{12}$. The vector $\mathbf{q}$ is often expressed in terms of an intensive variable through a constitutive relation. ${ }^{\mathrm{d}}$ The flux, then, conveys a notion of direction out of and into a region and is a proxy for the interaction between $\Omega_{1}$ and $\Omega_{2}$. It is important to note that the flux from $\Omega_{1}$ into $\Omega_{2}$ occurs across their common boundary and that if the two disjoint regions have no common boundary, then the flux from one to the other is zero. The classical flux (2.1) is then deemed to be local because there is no interaction between $\Omega_{1}$ and $\Omega_{2}$ when separated by a finite distance. The classical flux satisfies the action-reaction principle ${ }^{\mathrm{e}}$

$$
\int_{\partial \Omega_{12}} \mathbf{q} \cdot \mathbf{n}_{1} d A+\int_{\partial \Omega_{21}} \mathbf{q} \cdot \mathbf{n}_{2} d A=0
$$

where, of course, $\partial \Omega_{12}=\partial \Omega_{21}$ and $\mathbf{n}_{2}=-\mathbf{n}_{1}$ denotes the unit normal on $\partial \Omega_{21}$ pointing outward from $\Omega_{2}$. In words, the flux $\int_{\partial \Omega_{12}} \mathbf{q} \cdot \mathbf{n}_{1} d A$ from $\Omega_{1}$ into $\Omega_{2}$ across their common boundary $\partial \Omega_{12}$ is equal and opposite to the flux $\int_{\partial \Omega_{21}} \mathbf{q} \cdot \mathbf{n}_{2} d A$ from $\Omega_{2}$ into $\Omega_{1}$ across that same surface.

\subsection{Nonlocal fluxes}

We identify

$$
\int_{\Omega_{1}} \int_{\Omega_{2}} \psi(\mathbf{x}, \mathbf{y}) d \mathbf{y} d \mathbf{x}
$$

as a scalar interaction, or nonlocal flux, from $\Omega_{1}$ into $\Omega_{2}$, where $\psi:\left(\Omega_{1} \cup \Omega_{2}\right) \times$ $\left(\Omega_{1} \cup \Omega_{2}\right) \rightarrow \mathbb{R}$ denotes an antisymmetric function. We have that $\int_{\Omega_{2}} \psi(\mathbf{x}, \mathbf{y}) d \mathbf{y}$ is the flux density into $\Omega_{2}$ from the point $\mathbf{x} \in \Omega_{1}$ and likewise, $\int_{\Omega_{1}} \psi(\mathbf{x}, \mathbf{y}) d \mathbf{x}$ is the flux density into $\Omega_{1}$ from the point $\mathbf{y} \in \Omega_{2}$. As is the case for the local flux density $\mathbf{q} \cdot \mathbf{n}_{1}$, the nonlocal flux density $\int_{\Omega_{2}} \psi(\mathbf{x}, \mathbf{y}) d \mathbf{y}$ is related to an intensive variable through a constitutive relation; see Section 7.1.2.

From (1.1), it is easily seen that the antisymmetry of $\psi(\mathbf{x}, \mathbf{y})$ is equivalent to the nonlocal action-reaction principle

$$
\int_{\Omega_{1}} \int_{\Omega_{2}} \psi(\mathbf{x}, \mathbf{y}) d \mathbf{y} d \mathbf{x}+\int_{\Omega_{2}} \int_{\Omega_{1}} \psi(\mathbf{x}, \mathbf{y}) d \mathbf{y} d \mathbf{x}=0 \quad \forall \Omega_{1}, \Omega_{2} \subset \mathbb{R}^{n} ;
$$

(2.4) is the nonlocal analogue of (2.2). In words, (2.4) states that the flux (or interaction) from $\Omega_{1}$ into $\Omega_{2}$ is equal and opposite to the flux (or interaction) from

${ }^{\mathrm{d}}$ For example, if $\mathbf{q} \cdot \mathbf{n}_{1}$ denotes the heat flux density, then $\mathbf{q}$ is related to the temperature via Fourier's heat law; see Section 7.1.1.

eAn example is in mechanics for which Newton's third law, i.e., the force exerted upon on object is equal and opposite to the force exerted by the object, is an action-reaction archtype. 
$\Omega_{2}$ into $\Omega_{1}$. The flux is nonlocal because, by (2.4), the interaction may be nonzero even when the closures of $\Omega_{1}$ and $\Omega_{2}$ have an empty intersection. This is in stark contrast to classical local interactions for which we have seen that the interaction between $\Omega_{1}$ and $\Omega_{2}$ vanishes if their closures have empty intersection, i.e., have no common boundary.

\section{Nonlocal operators}

The nonlocal vector calculus developed in Section 4 involves nonlocal operators that mimic the classical local differential divergence, gradient, and curl operators. An important distinction between local and nonlocal operators is that the adjoint operators for the former involve the same operators, i.e., the adjoint of $\nabla \cdot$ is $-\nabla$, of $\nabla$ is $-\nabla \cdot$, and of $\nabla \times$ is $\nabla \times$, whereas the adjoint of nonlocal operators involve differently defined nonlocal gradient, divergence, and curl operators.

At this point, the association of the nonlocal operators given in Definition 3.1 and Theorem 3.1 with the operators of the classical differential vector calculus is purely conjectural. Justifications for making these associations are provided in Section 5 .

\subsection{Nonlocal point divergence, gradient, and curl operators}

The nonlocal point divergence, gradient, and curl operators map two-point functions to point functions and are defined in terms of their action on two-point functions as follows. These operators along with their adjoints are the building blocks of our nonlocal calculus.

Definition 3.1. [Nonlocal operators] Given the vector two-point function $\boldsymbol{\nu}: \mathbb{R}^{n} \times \mathbb{R}^{n} \rightarrow \mathbb{R}^{k}$ and the antisymmetric vector two-point function $\boldsymbol{\alpha}: \mathbb{R}^{n} \times \mathbb{R}^{n} \rightarrow$ $\mathbb{R}^{k}$, the action of the nonlocal point divergence operator $\mathcal{D}$ on $\boldsymbol{\nu}$ is defined as

$$
\mathcal{D}(\boldsymbol{\nu})(\mathbf{x}):=\int_{\mathbb{R}^{n}}\left(\boldsymbol{\nu}+\boldsymbol{\nu}^{\prime}\right) \cdot \boldsymbol{\alpha} d \mathbf{y} \quad \text { for } \mathbf{x} \in \mathbb{R}^{n}
$$

where $\mathcal{D}(\boldsymbol{\nu}): \mathbb{R}^{n} \rightarrow \mathbb{R}$. Given the scalar two-point function $\eta: \mathbb{R}^{n} \times \mathbb{R}^{n} \rightarrow \mathbb{R}$ and the antisymmetric vector two-point function $\boldsymbol{\beta}: \mathbb{R}^{n} \times \mathbb{R}^{n} \rightarrow \mathbb{R}^{k}$, the action of the nonlocal point gradient operator $\mathcal{G}$ on $\eta$ is defined as

$$
\mathcal{G}(\eta)(\mathbf{x}):=\int_{\mathbb{R}^{n}}\left(\eta+\eta^{\prime}\right) \boldsymbol{\beta} d \mathbf{y} \quad \text { for } \mathbf{x} \in \mathbb{R}^{n},
$$

where $\mathcal{G}(\eta): \mathbb{R}^{n} \rightarrow \mathbb{R}^{k}$. Given the vector two-point function $\boldsymbol{\mu}: \mathbb{R}^{n} \times \mathbb{R}^{n} \rightarrow \mathbb{R}^{3}$ and the antisymmetric vector two-point function $\gamma: \mathbb{R}^{n} \times \mathbb{R}^{n} \rightarrow \mathbb{R}^{3}$, the action of the nonlocal point curl operator $\mathcal{C}$ on $\boldsymbol{\mu}$ is defined as

$$
\mathcal{C}(\boldsymbol{\mu})(\mathbf{x}):=\int_{\mathbb{R}^{n}} \boldsymbol{\gamma} \times\left(\boldsymbol{\mu}+\boldsymbol{\mu}^{\prime}\right) d \mathbf{y} \quad \text { for } \mathbf{x} \in \mathbb{R}^{n},
$$

where $\mathcal{C}(\boldsymbol{\mu}): \mathbb{R}^{n} \rightarrow \mathbb{R}^{3}$. 
The nonlocal point operators $\mathcal{D}, \mathcal{G}$, and $\mathcal{C}$ map vectors to scalars, scalars to vectors, and vectors to vectors, respectively, as is the case for the divergence, gradient, and curl differential operators. Relationships between the nonlocal point operators and differential operators are made in Section 5 where we demonstrate circumstances under which the nonlocal point operators are identified with the corresponding differential operators in the sense of distributions and also as weak representations.

Because the integrands in (3.1a)-(3.1c) are antisymmetric, (1.1) immediately implies that

$$
\int_{\mathbb{R}^{n}} \mathcal{D}(\boldsymbol{\nu}) d \mathbf{x}=0, \quad \int_{\mathbb{R}^{n}} \mathcal{G}(\eta) d \mathbf{x}=\mathbf{0}, \quad \text { and } \quad \int_{\mathbb{R}^{n}} \mathcal{C}(\boldsymbol{\mu}) d \mathbf{x}=\mathbf{0} .
$$

These relations may be viewed as free-space nonlocal integral theorems. ${ }^{\mathrm{f}}$

\subsection{Nonlocal adjoint operators}

The adjoint operators corresponding to the nonlocal point operators are two-point operators that are defined as follows.

Definition 3.2. Given a point operator $\mathcal{Q}$ that maps two-point functions $F$ to point functions defined over $\mathbb{R}^{n}$, the adjoint operator $\mathcal{Q}^{*}$ is a two-point operator that maps point functions $G$ to two-point functions defined over $\mathbb{R}^{n} \times \mathbb{R}^{n}$ that satisfies

$$
(G, \mathcal{Q}(F))_{\mathbb{R}^{n}}-\left(\mathcal{Q}^{*}(G), F\right)_{\mathbb{R}^{n} \times \mathbb{R}^{n}}=0,
$$

where, for $\mathcal{Q}=\mathcal{D}, \mathcal{G}$, or $\mathcal{C}$, we have that $F$ and $G$ denote pairs of vector-scalar, scalar-vector, or vector-vector functions, respectively.

Definition 3.3 can be used to determine the nonlocal adjoint two-point operators corresponding to the nonlocal point operators introduced in Definition 3.1.

Theorem 3.1. [Nonlocal adjoint operators] Given the point function $u: \mathbb{R}^{n}$ $\rightarrow \mathbb{R}$, the adjoint of $\mathcal{D}$ is the two-point operator whose action on $u$ is given by

$$
\mathcal{D}^{*}(u)(\mathbf{x}, \mathbf{y})=-\left(u^{\prime}-u\right) \boldsymbol{\alpha} \quad \text { for } \mathbf{x}, \mathbf{y} \in \mathbb{R}^{n},
$$

where $\mathcal{D}^{*}(u): \mathbb{R}^{n} \times \mathbb{R}^{n} \rightarrow \mathbb{R}^{k}$. Given the point function $\mathbf{v}: \mathbb{R}^{n} \rightarrow \mathbb{R}^{k}$, the adjoint of $\mathcal{G}$ is the two-point operator whose action on $\mathbf{v}$ is given by

$$
\mathcal{G}^{*}(\mathbf{v})(\mathbf{x}, \mathbf{y})=-\left(\mathbf{v}^{\prime}-\mathbf{v}\right) \cdot \boldsymbol{\beta} \quad \text { for } \mathbf{x}, \mathbf{y} \in \mathbb{R}^{n},
$$

where $\mathcal{G}^{*}(\mathbf{v}): \mathbb{R}^{n} \times \mathbb{R}^{n} \rightarrow \mathbb{R}$. Given the point function $\mathbf{w}: \mathbb{R}^{n} \rightarrow \mathbb{R}^{3}$, the adjoint of $\mathcal{C}$ is the two-point operator whose action on $\mathbf{w}$ is given by

$$
\mathcal{C}^{*}(\mathbf{w})(\mathbf{x}, \mathbf{y})=\gamma \times\left(\mathbf{w}^{\prime}-\mathbf{w}\right) \quad \text { for } \mathbf{x}, \mathbf{y} \in \mathbb{R}^{n},
$$

${ }^{\mathrm{f}}$ For example, $\int_{\mathbb{R}^{n}} \mathcal{D}(\boldsymbol{\nu}) d \mathbf{x}=0$ can be viewed as a nonlocal analog of the free space classical local Gauss theorem $\int_{\mathbb{R}^{n}} \nabla \cdot \mathbf{u} d \mathbf{x}=0$. 
where $\mathcal{C}^{*}(\mathbf{w}): \mathbb{R}^{n} \times \mathbb{R}^{n} \rightarrow \mathbb{R}^{3}$.

Proof. Let $\boldsymbol{\xi}=u \boldsymbol{\nu}$. Then,

$$
\left(\boldsymbol{\xi}+\boldsymbol{\xi}^{\prime}\right) \cdot \boldsymbol{\alpha}=\left(u \boldsymbol{\nu}+u^{\prime} \boldsymbol{\nu}^{\prime}\right) \cdot \boldsymbol{\alpha}=u\left(\boldsymbol{\nu}+\boldsymbol{\nu}^{\prime}\right) \cdot \boldsymbol{\alpha}+\left(u^{\prime}-u\right) \boldsymbol{\nu}^{\prime} \cdot \boldsymbol{\alpha}
$$

so that, from (3.1a) and the first equation in (3.2), we have

$$
\begin{aligned}
0=\int_{\mathbb{R}^{n}} \mathcal{D}(\boldsymbol{\xi}) d \mathbf{x} & =\int_{\mathbb{R}^{n}} \int_{\mathbb{R}^{n}}\left(u\left(\boldsymbol{\nu}+\boldsymbol{\nu}^{\prime}\right) \cdot \boldsymbol{\alpha}+\left(u^{\prime}-u\right) \boldsymbol{\nu}^{\prime} \cdot \boldsymbol{\alpha}\right) d \mathbf{y} d \mathbf{x} \\
& =\int_{\mathbb{R}^{n}} u \int_{\mathbb{R}^{n}}\left(\boldsymbol{\nu}+\boldsymbol{\nu}^{\prime}\right) \cdot \boldsymbol{\alpha} d \mathbf{y} d \mathbf{x}+\int_{\mathbb{R}^{n}} \int_{\mathbb{R}^{n}}\left(u^{\prime}-u\right) \boldsymbol{\nu}^{\prime} \cdot \boldsymbol{\alpha} d \mathbf{y} d \mathbf{x} \\
& =\int_{\mathbb{R}^{n}} u \mathcal{D}(\boldsymbol{\nu}) d \mathbf{x}+\int_{\mathbb{R}^{n}} \int_{\mathbb{R}^{n}}\left(u^{\prime}-u\right) \boldsymbol{\nu} \cdot \boldsymbol{\alpha} d \mathbf{y} d \mathbf{x},
\end{aligned}
$$

where the last equality follows because we have, due to the antisymmetry of $\boldsymbol{\alpha}$, that $\left(u^{\prime}-u\right)\left(\boldsymbol{\nu}-\boldsymbol{\nu}^{\prime}\right) \cdot \boldsymbol{\alpha}$ is an antisymmetric two-point function so that, by (1.1),

$$
\int_{\mathbb{R}^{n}} \int_{\mathbb{R}^{n}}\left(u^{\prime}-u\right)\left(\boldsymbol{\nu}-\boldsymbol{\nu}^{\prime}\right) \cdot \boldsymbol{\alpha} d \mathbf{y} d \mathbf{x}=0
$$

'Associating $F$ with $\boldsymbol{\nu}, G$ with $u$, and $\mathcal{Q}$ with $\mathcal{D}$, we see that (3.6) is exactly of the form (3.3) with $\mathcal{Q}^{*}=\mathcal{D}^{*}$, where $\mathcal{D}^{*}$ is given by (3.4a).

In a similar manner, $(3.4 \mathrm{~b})$ can be derived from $(3.1 \mathrm{~b})$ and the second equation in (3.2) and (3.4c) can be derived from (3.1c) and the third equation in (3.2).

\subsection{Further observations and results about nonlocal operators}

In this subsection, we collect several observations and results that can be deduced from the definitions and results of Sections 3.1 and 3.2.

3.3.1. A nonlocal divergence operator for tensor functions and a nonlocal gradient operator for vector functions

A nonlocal divergence operator for tensor functions is defined by applying (3.1a) to each row of the tensor.

Definition 3.3. Given the tensor two-point function $\Psi: \mathbb{R}^{n} \times \mathbb{R}^{n} \rightarrow \mathbb{R}^{m \times k}$ and the antisymmetric vector two-point function $\boldsymbol{\alpha}: \mathbb{R}^{n} \times \mathbb{R}^{n} \rightarrow \mathbb{R}^{k}$, the action of the nonlocal point divergence operator $\mathcal{D}_{t}$ for tensors on $\boldsymbol{\Psi}$ is defined as

$$
\mathcal{D}_{t}(\Psi)(\mathbf{x}):=\int_{\mathbb{R}^{n}}\left(\Psi+\Psi^{\prime}\right) \cdot \boldsymbol{\alpha} d \mathbf{y} \quad \text { for } \mathbf{x} \in \mathbb{R}^{n},
$$

where $\mathcal{D}_{t}(\mathbf{\Psi})(\mathbf{x}): \mathbb{R}^{n} \rightarrow \mathbb{R}^{m}$.

Definition 3.2 implies that, given the point function $\mathbf{v}(\mathbf{x}): \mathbb{R}^{n} \rightarrow \mathbb{R}^{m}$, the action of the adjoint of $\mathcal{D}_{t}$ on $\mathbf{v}$ is given by

$$
\mathcal{D}_{t}^{*}(\mathbf{v})(\mathbf{x}, \mathbf{y})=-\left(\mathbf{v}^{\prime}-\mathbf{v}\right) \otimes \boldsymbol{\alpha} \quad \text { for } \mathbf{x}, \mathbf{y} \in \mathbb{R}^{n},
$$


where $\mathcal{D}_{t}^{*}(\mathbf{v}): \mathbb{R}^{n} \times \mathbb{R}^{n} \rightarrow \mathbb{R}^{m \times k}$, i.e., $\mathcal{D}_{t}^{*}$ maps a vector point function to a secondorder tensor two-point function. ${ }^{\mathrm{g}}$

One can also extend Definition (3.1b) of the nonlocal gradient operator of twopoint scalar functions to define the nonlocal gradient operator $\mathcal{G}_{v}$ acting on a twopoint vector function $\psi(\mathbf{x}, \mathbf{y})$ as the point tensor function

$$
\mathcal{G}_{v}(\boldsymbol{\psi})(\mathbf{x})=\int_{\mathbb{R}^{n}}\left(\boldsymbol{\psi}^{\prime}+\boldsymbol{\psi}\right) \otimes \boldsymbol{\beta} d \mathbf{y}
$$

The corresponding nonlocal adjoint operator $\mathcal{G}_{v}^{*}$ acting on a point tensor function $\mathbf{U}(\mathbf{x})$ is then given by the two-point vector function

$$
\mathcal{G}_{v}^{*}(\mathbf{U})(\mathbf{x}, \mathbf{y})=-\left(\mathbf{U}^{\prime}-\mathbf{U}\right) \cdot \boldsymbol{\beta} .
$$

\subsubsection{Nonlocal vector identities}

Compositions of the point operators defined in Sections 3.1 and 3.3.1 with the corresponding adjoint two-point operators derived in Sections 3.2 and 3.3.1 lead to the following nonlocal vector identities. In this proposition, we set $\boldsymbol{\alpha}=\boldsymbol{\beta}=\boldsymbol{\gamma}$ and, for the first, second, and fourth results, i.e., those involving nonlocal curl operators, we set $m=k=3$; we also set $m=k$ for the third result.

Proposition 3.1. The nonlocal divergence, gradient, and curl operators and the corresponding adjoint operators satisfy ${ }^{\mathrm{h}}$

$$
\begin{aligned}
& \mathcal{D}\left(\mathcal{C}^{*}(\mathbf{u})\right)=0 \quad \text { for } \mathbf{u}: \mathbb{R}^{n} \rightarrow \mathbb{R}^{3} \\
& \mathcal{C}\left(\mathcal{D}^{*}(u)\right)=\mathbf{0} \quad \text { for } u: \mathbb{R}^{n} \rightarrow \mathbb{R} \\
& \mathcal{G}^{*}(\mathbf{u})=\operatorname{tr}\left(\mathcal{D}_{t}^{*}(\mathbf{u})\right) \quad \text { for } \mathbf{u}: \mathbb{R}^{n} \rightarrow \mathbb{R}^{k} \\
& \mathcal{D}_{t}\left(\mathcal{D}_{t}^{*}(\mathbf{u})\right)-\mathcal{G}\left(\mathcal{G}^{*}(\mathbf{u})\right)=\mathcal{C}\left(\mathcal{C}^{*}(\mathbf{u})\right) \quad \text { for } \mathbf{u}: \mathbb{R}^{n} \rightarrow \mathbb{R}^{3} \text {. }
\end{aligned}
$$

${ }^{g}$ We could, in a slight abuse of notation, abbreviate $\mathcal{D}_{t}(\Psi)$ by $\mathcal{D}(\Psi)$. In an analogous fashion, $\mathcal{D}_{t}^{*}(\mathbf{v})$ may be abbreviated by $\mathcal{D}^{*}(\mathbf{v})$ when the argument is a vector point function. Note that this notational abuse is customary for the differential divergence and gradient operators for which $\nabla$. is used to denote the divergence operator for both vectors and tensors and $\nabla$ is used to denote the gradient operator on both scalars and vectors.

${ }^{\mathrm{h}}$ The four identities in (3.9) are analogous to the vector identities associated with the differential divergence, gradient and curl operator:

$$
\begin{aligned}
& \nabla \cdot(\nabla \times \mathbf{u})=0, \quad \nabla \times(\nabla u)=\mathbf{0}, \quad \nabla \cdot \mathbf{u}=\operatorname{tr}(\nabla \mathbf{u}), \\
& -\nabla \cdot(\nabla \mathbf{u})+\nabla(\nabla \cdot \mathbf{u})=\nabla \times(\nabla \times \mathbf{u}),
\end{aligned}
$$

respectively. Because $(\nabla \cdot)^{*}=-\nabla, \nabla^{*}=-\nabla \cdot$, and $(\nabla \times)^{*}=(\nabla \times)$, these identities can be written in the form

$$
\begin{aligned}
& \left.\left.\nabla \cdot\left((\nabla \times)^{*} \mathbf{u}\right)\right)=0, \quad \nabla \times\left((\nabla \cdot)^{*} \mathbf{u}\right)\right)=\mathbf{0}, \quad \nabla^{*} \mathbf{u}=\operatorname{tr}\left((\nabla \cdot)^{*} \mathbf{u}\right), \\
& \nabla \cdot\left((\nabla \cdot)^{*} \mathbf{u}\right)-\nabla\left((\nabla)^{*} \mathbf{u}\right)=\nabla \times\left((\nabla \times)^{*} \mathbf{u}\right)
\end{aligned}
$$

that more directly correspond to (3.9a)-(3.9d), respectively. In particular, the identities (3.9a), $(3.9 \mathrm{~b})$, and $(3.9 \mathrm{~d})$ suggest that $-\mathcal{D}^{*},-\mathcal{G}^{*}$, and $\mathcal{C}^{*}$ can also be viewed as nonlocal analogs of the differential gradient, divergence, and curl operators, respectively, that, when operating on point functions, result in two-point functions. 
Proof. We prove (3.9d); the proofs of $(3.9 \mathrm{a})-(3.9 \mathrm{c})$ are immediate after direct substitution of the operators involved.

Let $\mathbf{u}: \mathbb{R}^{n} \rightarrow \mathbb{R}^{3}$. Then, by (3.1c), (3.4c), (3.8a), and (3.8b) and recalling that $\boldsymbol{\alpha}$ is an antisymmetric function, we have

$$
\begin{aligned}
\mathcal{D}_{t}\left(\mathcal{D}_{t}^{*}(\mathbf{u})\right)-\mathcal{G}\left(\mathcal{G}^{*}(\mathbf{u})\right)= & -\int_{\mathbb{R}^{n}}\left(\left(\mathbf{u}^{\prime}-\mathbf{u}\right) \otimes \boldsymbol{\alpha}+\left(\mathbf{u}-\mathbf{u}^{\prime}\right) \otimes \boldsymbol{\alpha}^{\prime}\right) \cdot \boldsymbol{\alpha} d \mathbf{y} \\
& +\int_{\mathbb{R}^{n}}\left(\left(\mathbf{u}^{\prime}-\mathbf{u}\right) \cdot \boldsymbol{\alpha}+\left(\mathbf{u}-\mathbf{u}^{\prime}\right) \cdot \boldsymbol{\alpha}^{\prime}\right) \boldsymbol{\alpha} d \mathbf{y} \\
= & -2 \int_{\mathbb{R}^{n}}\left(\left(\mathbf{u}^{\prime}-\mathbf{u}\right)(\boldsymbol{\alpha} \cdot \boldsymbol{\alpha})-\left(\left(\mathbf{u}^{\prime}-\mathbf{u}\right) \cdot \boldsymbol{\alpha}\right) \boldsymbol{\alpha}\right) d \mathbf{y} \\
= & -2 \int_{\mathbb{R}^{n}}\left(\boldsymbol{\alpha} \times\left(\left(\mathbf{u}^{\prime}-\mathbf{u}\right) \times \boldsymbol{\alpha}\right)\right) d \mathbf{y},
\end{aligned}
$$

where, for the last equality, we have used the vector identity $\mathbf{a} \times(\mathbf{b} \times \mathbf{c})=\mathbf{b}(\mathbf{c} \cdot \mathbf{a})-$ $\mathbf{c}(\mathbf{a} \cdot \mathbf{b})$. A simple computation shows that the last expression is equal to $\mathcal{C}\left(\mathcal{C}^{*}(\mathbf{u})\right)$ so that $(3.9 \mathrm{~d})$ is proved.

Functions of the form $\mathcal{C}^{*}(\mathbf{u})$ do not entirely comprise the null space of the operator $\mathcal{D}$. In fact, it is obvious that for any antisymmetric two-point function $\boldsymbol{\nu}(\mathbf{x}, \mathbf{y})$, we have $\mathcal{D}(\boldsymbol{\nu})=0$. However, functions of the form $\mathcal{C}^{*}(\mathbf{u})$ are the only symmetric two-point functions belonging to the null space of $\mathcal{D}$. Analogous statements can be made for the null space of the operator $\mathcal{C}$ and two-point functions of the form $\mathcal{D}^{*}(u)$. Note that, because of the nonlocality of the operators, $\mathcal{G}^{*}(\mathcal{C}(\boldsymbol{\mu})) \neq 0$ and $\mathcal{C}^{*}(\mathcal{G}(\eta)) \neq 0$.

Another set of results for the nonlocal operators that mimic obvious properties of the corresponding differential operators are given in the following proposition whose proof is a straightforward consequence of the definitions given in Theorem 3.1 .

Proposition 3.2. Let $b$ and $\mathbf{b}$ denote a constant scalar and vector, respectively. Then, the adjoints of the nonlocal divergence, gradient, and curl operators satisfy

$$
\mathcal{D}^{*}(b)=0, \quad \mathcal{G}^{*}(\mathbf{b})=\mathbf{0}, \quad \text { and } \quad \mathcal{C}^{*}(\mathbf{b})=\mathbf{0} .
$$

These results do not hold for the point divergence, gradient, and curl operators, i.e., $\mathcal{D}(\mathbf{b}), \mathcal{G}(b)$, and $\mathcal{C}(\mathbf{b})$ do not necessarily vanish for constants $b$ and constant vectors $\mathbf{b}$.

\subsubsection{Nonlocal curl operators in two and higher dimensions}

The nonlocal point and two-point curl operators defined in (3.1c) and (3.4c), respectively, were defined in three dimensions. These definitions can be generalized to arbitrary dimensions by replacing the vector cross product with the wedge product so that, e.g., instead of (3.4c) we would have $\mathcal{C}^{*}(\mathbf{w}): \mathbb{R}^{n} \times \mathbb{R}^{n} \rightarrow \mathbb{R}^{r}$ given by

$$
\mathcal{C}^{*}(\mathbf{w})(\mathbf{x}, \mathbf{y})=\gamma \wedge\left(\mathbf{w}^{\prime}-\mathbf{w}\right),
$$


where $\gamma(\mathbf{x}, \mathbf{y}): \mathbb{R}^{n} \times \mathbb{R}^{n} \rightarrow \mathbb{R}^{r}$ with $r$ a positive integer. This suggests a possible exterior calculus-based formalism. However, such a formalism is beyond the scope of this paper so that only the special cases of $r=3$ and $r=2$ are considered and the vector cross product is retained.

We define nonlocal point and two-point curl operators in two space dimensions, i.e., for $\mathbb{R}^{2}$; in fact, we have two types of each kind of curl operator. ${ }^{i}$ First, we assume, without loss of generality, that $\boldsymbol{\mu} \cdot \mathbf{e}_{3}=0, \mathbf{w} \cdot \mathbf{e}_{3}=0$, and $\boldsymbol{\gamma} \cdot \mathbf{e}_{3}=0$ in (3.1c) and (3.4c). Then, for a vector two-point function $\boldsymbol{\mu}(\mathbf{x}, \mathbf{y}): \mathbb{R}^{n} \times \mathbb{R}^{n} \rightarrow \mathbb{R}^{2}$, we can view $\mathcal{C}(\boldsymbol{\mu})$ as the nonlocal scalar point function defined by

$$
\mathcal{C}(\boldsymbol{\mu})(\mathbf{x})=\int_{\mathbb{R}^{n}}\left(\left(\mu_{2}+\mu_{2}^{\prime}\right) \gamma_{1}-\left(\mu_{1}+\mu_{1}^{\prime}\right) \gamma_{2}\right) d \mathbf{y} \quad \text { for } \mathbf{x} \in \mathbb{R}^{n}
$$

and, for a vector point function $\mathbf{w}: \mathbb{R}^{n} \rightarrow \mathbb{R}^{2}$, we can view $\mathcal{C}^{*}(\mathbf{w})$ as the nonlocal scalar two-point function defined by

$$
\mathcal{C}^{*}(\mathbf{w})(\mathbf{x}, \mathbf{y})=\left(w_{2}^{\prime}-w_{2}\right) \gamma_{1}-\left(w_{1}^{\prime}-w_{1}\right) \gamma_{2} \quad \text { for } \mathbf{x}, \mathbf{y} \in \mathbb{R}^{n} .
$$

Next, assume, again without loss of generality, that $\boldsymbol{\mu}=\mu \mathbf{e}_{3}, \mathbf{w}=w \mathbf{e}_{3}$, and $\boldsymbol{\gamma} \cdot \mathbf{e}_{3}=0$ in (3.1c) and (3.4c). Then, for a scalar two-point function $\mu(\mathbf{x}, \mathbf{y}): \mathbb{R}^{n} \times \mathbb{R}^{n} \rightarrow \mathbb{R}$, we can view $\mathcal{C}(\mu)$ as the nonlocal vector point function defined by

$$
\mathcal{C}(\mu)(\mathbf{x})=\int_{\mathbb{R}^{n}}\left(\mu+\mu^{\prime}\right)\left(\gamma_{2} \mathbf{e}_{1}-\gamma_{1} \mathbf{e}_{2}\right) d \mathbf{y} \quad \text { for } \mathbf{x} \in \mathbb{R}^{n}
$$

and, for a scalar point function $w(\mathbf{x}): \mathbb{R}^{n} \rightarrow \mathbb{R}$, we can view $\mathcal{C}^{*}(w)$ as the nonlocal vector two-point function defined by

$$
\mathcal{C}^{*}(w)(\mathbf{x}, \mathbf{y})=\left(w^{\prime}-w\right)\left(\gamma_{2} \mathbf{e}_{1}-\gamma_{1} \mathbf{e}_{2}\right) \quad \text { for } \mathbf{x}, \mathbf{y} \in \mathbb{R}^{n} .
$$

\section{A nonlocal vector calculus}

We develop a nonlocal vector calculus that mimics the classical vector calculus for differential operators. In the classical calculus, interactions are local which is why, e.g., in the Gauss theorem, the contribution coming from interactions with points outside a domain $\Omega$ appears in the form of a flux through the boundary $\partial \Omega$. If interactions are nonlocal, then points outside of $\bar{\Omega}$ interact with points in $\Omega$ so that that interaction cannot be accounted for merely by an integral along the boundary $\partial \Omega$. In fact, one must account for interactions with points in the complement domain $\mathbb{R}^{n} \backslash \Omega$.

${ }^{i}$ This is analogous to the two types of differential curl operators in two dimensions, one operating on vectors, the other on scalars, respetively given by

$$
\operatorname{curl} \mathbf{u}=\frac{\partial u_{1}}{\partial x_{2}}-\frac{\partial u_{2}}{\partial x_{1}} \quad \text { and } \quad \operatorname{curl} u=-\frac{\partial u}{\partial x_{2}} \mathbf{e}_{1}+\frac{\partial u}{\partial x_{1}} \mathbf{e}_{2} .
$$


To treat the most general case, we define, for a given open subset $\Omega_{s} \subset \mathbb{R}^{n}$, the corresponding interaction domain

$$
\Omega_{c}:=\left\{\mathbf{y} \in \mathbb{R}^{n} \backslash \Omega_{s} \text { such that } \quad \boldsymbol{\alpha}(\mathbf{x}, \mathbf{y}) \neq \mathbf{0} \quad \text { for some } \mathbf{x} \in \Omega_{s}\right\}
$$

so that $\Omega_{c}$ consists of those points outside of $\Omega_{s}$ that interact with points in $\Omega_{s}$. For simplicity, we assume that the corresponding interactions domains for $\boldsymbol{\beta}$ and $\boldsymbol{\gamma}$ are also given by (4.1). No assumption is made about the geometric relation between $\Omega_{s}$ and $\Omega_{c}$ so that, e.g., the four configurations of Figure 1 are possible. Note that the situation $\Omega_{c}=\mathbb{R}^{n} \backslash \Omega_{s}$ is allowable as is $\Omega_{s}=\mathbb{R}^{n}$.
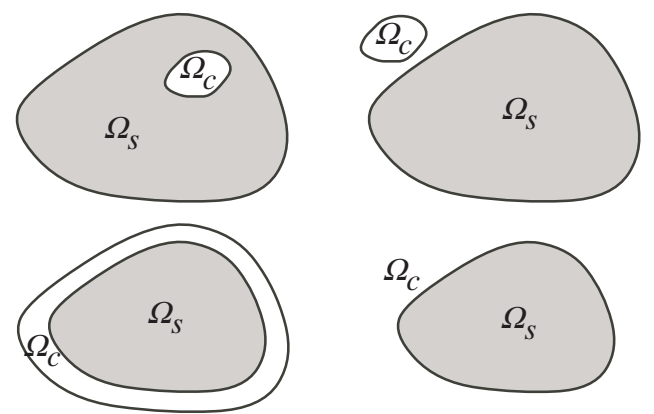

Fig. 1. Four of the possible configurations for $\Omega_{s}$ and $\Omega_{c}$.

We set $\Omega_{s c}=\Omega_{s} \cup \Omega_{c}$. From (4.1) we see that points in $\mathbb{R}^{n} \backslash \Omega_{s c}$ do not interact with points in $\Omega_{s}$, i.e.,

$$
\boldsymbol{\alpha}(\mathbf{x}, \mathbf{y})=\mathbf{0} \quad \text { for } \mathbf{x} \in \Omega_{s} \text { and } \mathbf{y} \in \mathbb{R}^{n} \backslash \Omega_{s c}
$$

and, by antisymmetry,

$$
\boldsymbol{\alpha}(\mathbf{x}, \mathbf{y})=\mathbf{0} \quad \text { for } \mathbf{y} \in \Omega_{s} \text { and } \mathbf{x} \in \mathbb{R}^{n} \backslash \Omega_{s c} .
$$

Note that we do not assume that points in $\Omega_{c}$ interact only with points in $\Omega_{s} \cup \Omega_{c}$, i.e., in general, points in $\Omega_{c}$ may interact with points in $\mathbb{R}^{n} \backslash \Omega_{s c}$ as well.

\subsection{Nonlocal interaction operators}

The nonlocal operators of Definition 3.1 describe the interaction of points in $\Omega_{s}$ with points not only in $\Omega_{s}$ but also with those in $\Omega_{c}$. Naturally, we need the same information for points in $\Omega_{c}$.

Definition 4.1. [Nonlocal interaction operators] Given a domain $\Omega_{s} \subset \mathbb{R}^{n}$, let the interaction domain $\Omega_{c}$ be defined by (4.1). Then, corresponding to the point divergence operator $\mathcal{D}(\boldsymbol{\nu}): \mathbb{R}^{n} \rightarrow \mathbb{R}$ defined in (3.1a), we define the action of the point interaction operator $\mathcal{N}(\boldsymbol{\nu}): \Omega_{s} \rightarrow \mathbb{R}$ on $\boldsymbol{\nu}$ by

$$
\mathcal{N}(\boldsymbol{\nu})(\mathbf{x}):=\int_{\Omega_{c}}\left(\boldsymbol{\nu}+\boldsymbol{\nu}^{\prime}\right) \cdot \boldsymbol{\alpha} d \mathbf{y} \quad \text { for } \mathbf{x} \in \Omega_{s} .
$$


Corresponding to the point gradient operator $\mathcal{G}(\eta): \mathbb{R}^{n} \rightarrow \mathbb{R}^{k}$ defined in (3.1b), we define the action of the point interaction operator $\mathcal{S}(\eta): \Omega_{s} \rightarrow \mathbb{R}^{k}$ on $\eta$ by

$$
\mathcal{S}(\eta)(\mathbf{x}):=\int_{\Omega_{c}}\left(\eta+\eta^{\prime}\right) \boldsymbol{\beta} d \mathbf{y} \quad \text { for } \mathbf{x} \in \Omega_{s} .
$$

Corresponding to the point curl operator $\mathcal{C}(\boldsymbol{\mu}): \mathbb{R}^{n} \rightarrow \mathbb{R}^{3}$ defined in (3.1c), we define the action of the point interaction operator $\mathcal{T}(\boldsymbol{\mu}): \Omega_{s} \rightarrow \mathbb{R}^{3}$ on $\boldsymbol{\mu}$ by

$$
\mathcal{T}(\boldsymbol{\mu})(\mathbf{x}):=\int_{\Omega_{c}} \boldsymbol{\gamma} \times\left(\boldsymbol{\mu}+\boldsymbol{\mu}^{\prime}\right) d \mathbf{y} \quad \text { for } \mathbf{x} \in \Omega_{s}
$$

Letting $\psi(\mathbf{x}, \mathbf{y})=\left(\boldsymbol{\nu}+\boldsymbol{\nu}^{\prime}\right) \cdot \boldsymbol{\alpha}$ and letting $\Omega_{1}=\Omega_{c}$ and $\Omega_{2}=\Omega_{s}$, we see from the discussion following $(2.3)$ that $\mathcal{N}(\boldsymbol{\nu})$ represents a nonlocal flux density into $\Omega_{c}$ from points in $\Omega_{s}$, and similarly for $\mathcal{S}(\eta)$ and $\mathcal{T}(\boldsymbol{\mu})$.

\subsection{Nonlocal integral theorems}

Definitions 3.1 and 4.1 lead to integral theorems for the nonlocal vector calculus that mimic the basic integral theorems of the vector calculus for differential operators.

Theorem 4.1. [Nonlocal integral theorems] Assuming the notations and definitions found in Definitions 3.1 and 4.1, we have

$$
\begin{aligned}
\int_{\Omega_{s}} \mathcal{D}(\boldsymbol{\nu}) d \mathbf{x} & =\int_{\Omega_{s}} \mathcal{N}(\boldsymbol{\nu}) d \mathbf{x} \\
\int_{\Omega_{s}} \mathcal{G}(\eta) d \mathbf{x} & =\int_{\Omega_{s}} \mathcal{S}(\eta) d \mathbf{x} \\
\int_{\Omega_{s}} \mathcal{C}(\boldsymbol{\mu}) d \mathbf{x} & =\int_{\Omega_{s}} \mathcal{T}(\boldsymbol{\mu}) d \mathbf{x} .
\end{aligned}
$$

Proof. We have that

$$
\begin{aligned}
\int_{\Omega_{s}} \mathcal{D}(\boldsymbol{\nu}) d \mathbf{x} & =\int_{\Omega_{s}} \int_{\mathbb{R}^{n}}\left(\boldsymbol{\nu}+\boldsymbol{\nu}^{\prime}\right) \cdot \boldsymbol{\alpha} d \mathbf{y} d \mathbf{x}=\int_{\Omega_{s}} \int_{\mathbb{R}^{n} / \Omega_{s}}\left(\boldsymbol{\nu}+\boldsymbol{\nu}^{\prime}\right) \cdot \boldsymbol{\alpha} d \mathbf{y} d \mathbf{x} \\
& =\int_{\Omega_{s}} \int_{\Omega_{c}}\left(\boldsymbol{\nu}+\boldsymbol{\nu}^{\prime}\right) \cdot \boldsymbol{\alpha} d \mathbf{y} d \mathbf{x}=\int_{\Omega_{s}} \mathcal{N}(\boldsymbol{\nu}) d \mathbf{x},
\end{aligned}
$$

where the four equalities follow in succession from (3.1a), the antisymmetry ${ }^{\mathrm{k}}$ of $\boldsymbol{\alpha}$ and (1.1), (4.1), and (4.2a). Thus, (4.3a) follows; (4.3b)and (4.3c) follow in exactly

${ }^{\mathrm{j}}$ The nonlocal integral theorems (4.3) are analogous to the classical differential integral theorems given by

$$
\int_{\Omega} \nabla \cdot \mathbf{v} d \mathbf{x}=\int_{\partial \Omega} \mathbf{v} \cdot \mathbf{n} d \mathbf{x}, \quad \int_{\Omega} \nabla v d \mathbf{x}=\int_{\partial \Omega} v \mathbf{n} d \mathbf{x}, \quad \text { and } \quad \int_{\Omega} \nabla \times \mathbf{v} d \mathbf{x}=\int_{\partial \Omega} \mathbf{n} \times \mathbf{v} d \mathbf{x}
$$

for functions $\mathbf{v}$ and $v$ defined on $\mathbb{R}^{n}$, with $n=3$ for the third one, for which the integrals are well defined.

${ }^{\mathrm{k}}$ Because $\boldsymbol{\alpha}$ is antisymmetric, the integrand in the double integral is antisymmetric, i.e., we have that $\left(\boldsymbol{\nu}+\boldsymbol{\nu}^{\prime}\right) \cdot \boldsymbol{\alpha}=-\left(\boldsymbol{\nu}^{\prime}+\boldsymbol{\nu}\right) \cdot \boldsymbol{\alpha}^{\prime}$. 
the same way starting with (3.1b) and (3.1c), respectively. Alternately, they can be derived from (4.3a); one simply chooses $\boldsymbol{\nu}=\eta \mathbf{b}$ and $\boldsymbol{\nu}=\mathbf{b} \times \boldsymbol{\mu}$, respectively, in that equation, where $\mathbf{b}$ is a constant vector; one also has to associate $\boldsymbol{\beta}$ and $\boldsymbol{\gamma}$ with $\boldsymbol{\alpha} \square$

In words, (4.3a) states that the integral of the nonlocal divergence of $\boldsymbol{\nu}$ over $\Omega_{s}$ is equal to the total flux out of $\Omega_{s}$ into $\Omega_{c}{ }^{1}{ }^{1}$ Similar interpretations of (4.3b) and (4.3c) hold.

The nonlocal integral theorems (4.3a)-(4.3c) are action-reaction principles. For example, from (4.3a) and the assumptions made, we have that

$$
\begin{aligned}
0 & =\int_{\Omega_{s}} \mathcal{N}(\boldsymbol{\nu}) d \mathbf{x}-\int_{\Omega_{s}} \mathcal{D}(\boldsymbol{\nu}) d \mathbf{x} \\
& =\int_{\Omega_{s}} \int_{\Omega_{c}}\left(\boldsymbol{\nu}+\boldsymbol{\nu}^{\prime}\right) \cdot \boldsymbol{\alpha} d \mathbf{y} d \mathbf{x}-\int_{\Omega_{s}} \int_{\mathbb{R}^{n}}\left(\boldsymbol{\nu}+\boldsymbol{\nu}^{\prime}\right) \cdot \boldsymbol{\alpha} d \mathbf{y} d \mathbf{x} \\
& =\int_{\Omega_{s}} \int_{\Omega_{c}}\left(\boldsymbol{\nu}+\boldsymbol{\nu}^{\prime}\right) \cdot \boldsymbol{\alpha} d \mathbf{y} d \mathbf{x}-\int_{\Omega_{s}} \int_{\Omega_{c}}\left(\boldsymbol{\nu}+\boldsymbol{\nu}^{\prime}\right) \cdot \boldsymbol{\alpha} d \mathbf{y} d \mathbf{x} \\
& =\int_{\Omega_{s}} \int_{\Omega_{c}}\left(\boldsymbol{\nu}+\boldsymbol{\nu}^{\prime}\right) \cdot \boldsymbol{\alpha} d \mathbf{y} d \mathbf{x}+\int_{\Omega_{c}} \int_{\Omega_{s}}\left(\boldsymbol{\nu}+\boldsymbol{\nu}^{\prime}\right) \cdot \boldsymbol{\alpha} d \mathbf{y} d \mathbf{x}
\end{aligned}
$$

which is exactly of the form (2.4) with $\Omega_{1}=\Omega_{s}, \Omega_{2}=\Omega_{c}$, and $\psi=\left(\boldsymbol{\nu}+\boldsymbol{\nu}^{\prime}\right) \cdot \boldsymbol{\alpha}$; (4.4) simply states that the flux out of $\Omega_{s}$ into $\Omega_{c}$ is equal and opposite to the flux from $\Omega_{c}$ into $\Omega_{s}$.

The nonlocal integral theorems (4.3a)-(4.3c) have the simple consequences listed in the following corollary that can be viewed as providing nonlocal integration by parts formulas.

Corollary 4.1. [Nonlocal integration by parts formulas] Adopt the hypotheses of Theorem 4.1 and let the nonlocal adjoint operators be given as in Theorem 3.1. Then, given the point functions $u(\mathbf{x}): \mathbb{R}^{n} \rightarrow \mathbb{R}, \mathbf{v}(\mathbf{x}): \mathbb{R}^{n} \rightarrow \mathbb{R}^{k}$, and

\footnotetext{
${ }^{1}$ This observation is analogous to the observation for the classical Gauss theorem $\int_{\Omega} \nabla \cdot \mathbf{u}=$ $\int_{\partial \Omega} \mathbf{u} \cdot \mathbf{n} d A$ that, by (2.1), the integral of the local divergence of $\mathbf{u}$ over $\Omega$ is equal to the total flux out of $\Omega$.
} 
$\mathbf{w}(\mathbf{x}): \mathbb{R}^{n} \rightarrow \mathbb{R}^{3}$, we have $e^{\mathrm{m}, \mathrm{n}}$

$$
\begin{gathered}
\int_{\Omega_{s}} u \mathcal{D}(\boldsymbol{\nu}) d \mathbf{x}-\int_{\Omega_{s}} \int_{\Omega_{s}} \mathcal{D}^{*}(u) \cdot \boldsymbol{\nu} d \mathbf{y} d \mathbf{x}=\int_{\Omega_{s}} u \mathcal{N}(\boldsymbol{\nu}) d \mathbf{x} \\
\int_{\Omega_{s}} \mathbf{v} \cdot \mathcal{G}(\eta) d \mathbf{x}-\int_{\Omega_{s}} \int_{\Omega_{s}} \mathcal{G}^{*}(\mathbf{v}) \eta d \mathbf{y} d \mathbf{x}=\int_{\Omega_{s}} \mathbf{v} \cdot \mathcal{S}(\eta) d \mathbf{x} \\
\int_{\Omega_{s}} \mathbf{w} \cdot \mathcal{C}(\boldsymbol{\mu}) d \mathbf{x}-\int_{\Omega_{s}} \int_{\Omega_{s}} \mathcal{C}^{*}(\mathbf{w}) \cdot \boldsymbol{\mu} d \mathbf{y} d \mathbf{x}=\int_{\Omega_{s}} \mathbf{w} \cdot \mathcal{T}(\boldsymbol{\mu}) d \mathbf{x}
\end{gathered}
$$

Proof. Let $\boldsymbol{\xi}=u \boldsymbol{\nu}$; then, from (3.1a) and (3.5), we have

$$
\begin{aligned}
\mathcal{D}(\boldsymbol{\xi}) & =\int_{\mathbb{R}^{n}}\left(u\left(\boldsymbol{\nu}+\boldsymbol{\nu}^{\prime}\right) \cdot \boldsymbol{\alpha}+\left(u^{\prime}-u\right) \boldsymbol{\nu}^{\prime} \cdot \boldsymbol{\alpha}\right) d \mathbf{y} \\
& =u \int_{\mathbb{R}^{n}}\left(\boldsymbol{\nu}+\boldsymbol{\nu}^{\prime}\right) \cdot \boldsymbol{\alpha} d \mathbf{y}+\int_{\mathbb{R}^{n}}\left(u^{\prime}-u\right) \boldsymbol{\nu}^{\prime} \cdot \boldsymbol{\alpha} d \mathbf{y} \\
& =u \mathcal{D}(\boldsymbol{\nu})+\int_{\mathbb{R}^{n}}\left(u^{\prime}-u\right) \boldsymbol{\nu}^{\prime} \cdot \boldsymbol{\alpha} d \mathbf{y} \\
& =u \mathcal{D}(\boldsymbol{\nu})+\int_{\Omega_{s c}}\left(u^{\prime}-u\right) \boldsymbol{\nu}^{\prime} \cdot \boldsymbol{\alpha} d \mathbf{y} \quad \forall \mathbf{x} \in \Omega_{s},
\end{aligned}
$$

where the last equality follows from (4.1). Also, from (4.2a), we have

$$
\begin{aligned}
\mathcal{N}(\boldsymbol{\xi}) & =\int_{\Omega_{c}}\left(u\left(\boldsymbol{\nu}+\boldsymbol{\nu}^{\prime}\right) \cdot \boldsymbol{\alpha}+\left(u^{\prime}-u\right) \boldsymbol{\nu}^{\prime} \cdot \boldsymbol{\alpha}\right) d \mathbf{y} \\
& =u \mathcal{N}(\boldsymbol{\nu})+\int_{\Omega_{c}}\left(u^{\prime}-u\right) \boldsymbol{\nu}^{\prime} \cdot \boldsymbol{\alpha} d \mathbf{y} \quad \forall \mathbf{x} \in \Omega_{s} .
\end{aligned}
$$

Then,

$$
\begin{aligned}
0= & \int_{\Omega_{s}} \mathcal{D}(\boldsymbol{\xi}) d \mathbf{x}-\int_{\Omega_{s}} \mathcal{N}(\boldsymbol{\xi}) d \mathbf{x} \\
= & \int_{\Omega_{s}} u \mathcal{D}(\boldsymbol{\nu}) d \mathbf{x}-\int_{\Omega_{s}} u \mathcal{N}(\boldsymbol{\nu}) d \mathbf{x} \\
& \quad+\int_{\Omega_{s}} \int_{\Omega_{s c}}\left(u^{\prime}-u\right) \boldsymbol{\nu}^{\prime} \cdot \boldsymbol{\alpha} d \mathbf{y} d \mathbf{x}-\int_{\Omega_{s}} \int_{\Omega_{c}}\left(u^{\prime}-u\right) \boldsymbol{\nu}^{\prime} \cdot \boldsymbol{\alpha} d \mathbf{y} d \mathbf{x} \\
= & \int_{\Omega_{s}} u \mathcal{D}(\boldsymbol{\nu}) d \mathbf{x}-\int_{\Omega_{c}} u \mathcal{N}(\boldsymbol{\nu}) d \mathbf{x}+\int_{\Omega_{s}} \int_{\Omega_{s}}\left(u^{\prime}-u\right) \boldsymbol{\nu}^{\prime} \cdot \boldsymbol{\alpha} d \mathbf{y} d \mathbf{x},
\end{aligned}
$$

${ }^{\mathrm{m}}$ If $\boldsymbol{\nu}$ and $\boldsymbol{\alpha}$ are scalar-valued functions and for free space, a version of the integration by parts formula (4.5a) appears in Lemma 2.1 of Ref. 10.

${ }^{\mathrm{n}}$ The classical analog of $(4.5 \mathrm{a})$ is given by, for a scalar function $u(\mathbf{x})$ and vector function $\mathbf{v}(\mathbf{x})$,

$$
\int_{\Omega} u \nabla \cdot \mathbf{v} d \mathbf{x}+\int_{\Omega} \mathbf{v} \cdot \nabla u d \mathbf{x}=\int_{\partial \Omega} u \mathbf{v} \cdot \mathbf{n} d A
$$

and similarly for (4.5b) and (4.5c). 
where the first equality follows from (4.3a), the second from (4.6) and (4.7), and the third from the linearity of the integration operator. Then, (4.5a) follows from the antisymmetry of $\boldsymbol{\alpha}$ and (3.7).

In a similar manner, (4.5b) and (4.5c) can be derived from (4.3a) along with (3.1b) and (3.1c), respectively. Alternately, (4.5b) and (4.5c) easily follow by setting, for an arbitrary constant vector $\mathbf{b}, \boldsymbol{\nu}=\eta \mathbf{b}$ and $\boldsymbol{\nu}=\mathbf{b} \times \boldsymbol{\mu}$, respectively, in (4.5a) and also associating $\boldsymbol{\beta}$ and $\boldsymbol{\gamma}$ with $\boldsymbol{\alpha}$.

\subsection{Nonlocal Green's identities}

Nonlocal (generalized) Green's identities are simple consequences of Corollary 4.1. For the following two corollaries, we carry over the notations, definitions, and results obtained above.

Corollary 4.2. [Nonlocal (generalized) Green's first identities] Given the scalar point functions $u(\mathbf{x}): \mathbb{R}^{n} \rightarrow \mathbb{R}$ and $v(\mathbf{x}): \mathbb{R}^{n} \rightarrow \mathbb{R}$ and the two-point secondorder tensor function $\mathbf{\Theta}(\mathbf{x}, \mathbf{y}): \mathbb{R}^{n} \times \mathbb{R}^{n} \rightarrow \mathbb{R}^{k \times k}$, then ${ }^{\circ}$

$$
\begin{aligned}
\int_{\Omega_{s}} u \mathcal{D}\left(\boldsymbol{\Theta} \cdot \mathcal{D}^{*}(v)\right) d \mathbf{x} & -\int_{\Omega_{s}} \int_{\Omega_{s}} \mathcal{D}^{*}(u) \cdot \boldsymbol{\Theta} \cdot \mathcal{D}^{*}(v) \boldsymbol{\nu} d \mathbf{y} d \mathbf{x} \\
& =\int_{\Omega_{s}} u \mathcal{N}\left(\boldsymbol{\Theta} \cdot \mathcal{D}^{*}(v)\right) d \mathbf{x} .
\end{aligned}
$$

Given the vector point functions $\mathbf{u}(\mathbf{x}): \mathbb{R}^{n} \rightarrow \mathbb{R}^{k}$ and $\mathbf{v}(\mathbf{x}): \mathbb{R}^{n} \rightarrow \mathbb{R}^{k}$ and the two-point scalar function $\theta(\mathbf{x}, \mathbf{y}): \mathbb{R}^{n} \times \mathbb{R}^{n} \rightarrow \mathbb{R}$, then

$$
\begin{aligned}
\int_{\Omega_{s}} \mathbf{v} \cdot \mathcal{G}\left(\theta \mathcal{G}^{*}(\mathbf{u})\right) d \mathbf{x} & -\int_{\Omega_{s}} \int_{\Omega_{s}} \theta \mathcal{G}^{*}(\mathbf{v}) \mathcal{G}^{*}(\mathbf{u}) d \mathbf{y} d \mathbf{x} \\
& =\int_{\Omega_{s}} \mathbf{v} \cdot \mathcal{S}\left(\theta \mathcal{G}^{*}(\mathbf{u})\right) d \mathbf{x} .
\end{aligned}
$$

Given the vector point functions $\mathbf{u}(\mathbf{x}): \mathbb{R}^{n} \rightarrow \mathbb{R}^{3}$ and $\mathbf{w}(\mathbf{x}): \mathbb{R}^{n} \rightarrow \mathbb{R}^{3}$ and the two-point second-order tensor function $\mathbf{\Theta}(\mathbf{x}, \mathbf{y}): \mathbb{R}^{n} \times \mathbb{R}^{n} \rightarrow \mathbb{R}^{3 \times 3}$, then

$$
\begin{aligned}
\int_{\Omega_{s}} \mathbf{w} \cdot \mathcal{C}\left(\boldsymbol{\Theta} \cdot \mathcal{C}^{*}(\mathbf{u})\right) d \mathbf{x} & -\int_{\Omega_{s}} \int_{\Omega_{s}} \mathcal{C}^{*}(\mathbf{w}) \cdot \boldsymbol{\Theta} \cdot \mathcal{C}^{*}(\mathbf{u}) d \mathbf{y} d \mathbf{x} \\
& =\int_{\Omega_{s}} \mathbf{w} \cdot \mathcal{T}\left(\boldsymbol{\Theta} \cdot \mathcal{C}^{*}(\mathbf{u})\right) d \mathbf{x} .
\end{aligned}
$$

${ }^{\circ}$ We have that (4.8a) and (4.9a) are the nonlocal analogs of the local classical (generalized) first Green's identity

$$
\int_{\Omega} u \nabla \cdot(\boldsymbol{\Theta} \cdot \nabla v) d \mathbf{x}+\int_{\Omega} \nabla u \cdot(\boldsymbol{\Theta} \nabla v) d \mathbf{x}=\int_{\partial \Omega} u \mathbf{n} \cdot(\boldsymbol{\Theta} \cdot \nabla v) d A
$$

and of the local classical (generalized) second Green's identity

$$
\int_{\Omega} u \nabla \cdot(\boldsymbol{\Theta} \cdot \nabla v) d \mathbf{x}-\int_{\Omega} v \nabla \cdot(\boldsymbol{\Theta} \cdot \nabla u) d \mathbf{x}=\int_{\partial \Omega} u \mathbf{n} \cdot(\boldsymbol{\Theta} \cdot \nabla u) d A-\int_{\partial \Omega} v \mathbf{n} \cdot(\boldsymbol{\Theta} \cdot \nabla u) d A,
$$

respectively, and similarly for $(4.8 \mathrm{~b}),(4.9 \mathrm{~b}),(4.8 \mathrm{c})$ and $(4.9 \mathrm{c})$. These are generalizations of the classical local Green's identities for which $\theta=1$ and $\Theta$ is the identity tensor. 
Proof. The results (4.8a)-(4.8c) follow by setting $\boldsymbol{\nu}=\boldsymbol{\Theta} \cdot \mathcal{D}^{*}(u)$ in (4.5a), $\eta=$ $\theta \mathcal{G}^{*}(\mathbf{u})$ in (4.5b), and $\boldsymbol{\mu}=\boldsymbol{\Theta} \cdot \mathcal{C}^{*}(u)$ in (4.5c), respectively.

Corollary 4.3. [Nonlocal (generalized) Green's second identities] We assume the same notation as in Corollary 4.2 and we also assume that the tensors $\boldsymbol{\Theta}$ appearing in (4.8a) and (4.8c) are symmetric. Then,

$$
\begin{gathered}
\int_{\Omega_{s}} u \mathcal{D}\left(\boldsymbol{\Theta} \cdot \mathcal{D}^{*}(v)\right) d \mathbf{x}-\int_{\Omega_{s}} v \mathcal{D}\left(\boldsymbol{\Theta} \cdot \mathcal{D}^{*}(u)\right) d \mathbf{x} \\
=\int_{\Omega_{s}} u \mathcal{N}\left(\boldsymbol{\Theta} \cdot \mathcal{D}^{*}(v)\right) d \mathbf{x}-\int_{\Omega_{s}} v \mathcal{N}\left(\boldsymbol{\Theta} \cdot \mathcal{D}^{*}(u)\right) d \mathbf{x} \\
\int_{\Omega_{s}} \mathbf{v} \cdot \mathcal{G}\left(\theta \mathcal{G}^{*}(\mathbf{u})\right) d \mathbf{x}-\int_{\Omega_{s}} \mathbf{u} \cdot \mathcal{G}\left(\theta \mathcal{G}^{*}(\mathbf{v})\right) d \mathbf{x} \\
=\int_{\Omega_{s}} \mathbf{v} \cdot \mathcal{S}\left(\theta \mathcal{G}^{*}(\mathbf{u})\right) d \mathbf{x}-\int_{\Omega_{s}} \mathbf{u} \cdot \mathcal{S}\left(\theta \mathcal{G}^{*}(\mathbf{v})\right) d \mathbf{x} \\
\int_{\Omega_{s}} \mathbf{w} \cdot \mathcal{C}\left(\boldsymbol{\Theta}: \mathcal{C}^{*}(\mathbf{u})\right) d \mathbf{x}-\int_{\Omega_{s}} \mathbf{u} \cdot \mathcal{C}\left(\boldsymbol{\Theta}: \mathcal{C}^{*}(\mathbf{w})\right) d \mathbf{x} \\
=\int_{\Omega_{s}} \mathbf{w} \cdot \mathcal{T}\left(\boldsymbol{\Theta}: \mathcal{C}^{*}(\mathbf{u})\right) d \mathbf{x}-\int_{\Omega_{s}} \mathbf{u} \cdot \mathcal{T}\left(\boldsymbol{\Theta}: \mathcal{C}^{*}(\mathbf{v})\right) d \mathbf{x} .
\end{gathered}
$$

Proof. The result (4.9a) is obtained by reversing the roles of $u$ and $v$ in (4.8a) and then subtracting the result from (4.8a). The results $(4.9 \mathrm{~b})$ and $(4.9 \mathrm{c})$ are obtained from $(4.8 \mathrm{~b})$ and $(4.8 \mathrm{c})$, respectively, in a similar manner.

\subsection{Special cases of the vector calculus}

We consider some special cases of the vector calculus we developed in Sections 4.1-4.3.

\subsubsection{The free space vector calculus}

As was mentioned previously, the vector calculus allows for the choice $\Omega_{s}=\mathbb{R}^{n}$ in which case we also simply set $\Omega_{c}=\emptyset$ in all definitions and results. The resulting nonlocal integral theorems have already been stated; see (3.2). Corresponding to the nonlocal divergence operator $\mathcal{D}$, we have the nonlocal generalized Green's first identity

$$
\int_{\mathbb{R}^{n}} u \mathcal{D}\left(\boldsymbol{\Theta} \cdot \mathcal{D}^{*}(v)\right) d \mathbf{x}-\int_{\mathbb{R}^{n}} \int_{\mathbb{R}^{n}} \mathcal{D}^{*}(u) \cdot \boldsymbol{\Theta} \cdot \mathcal{D}^{*}(v) \boldsymbol{\nu} d \mathbf{y} d \mathbf{x}=0
$$

and the generalized nonlocal Green's second identity

$$
\int_{\mathbb{R}^{n}} u \mathcal{D}\left(\boldsymbol{\Theta} \cdot \mathcal{D}^{*}(v)\right) d \mathbf{x}-\int_{\mathbb{R}^{n}} v \mathcal{D}\left(\boldsymbol{\Theta} \cdot \mathcal{D}^{*}(u)\right) d \mathbf{x}=0
$$

corresponding to (4.8a) and (4.9a), respectively, and similarly for the other operators. 


\subsubsection{The vector calculus for interactions of infinite extent}

In case points in $\Omega_{s}$ interact with all points in $\mathbb{R}^{n}$, we have that $\Omega_{c}=\mathbb{R}^{n} \backslash \Omega_{s}$. In this case, all definitions and results remain unchanged.

\subsubsection{The vector calculus for localized kernels}

An important special case is that of localized kernels for which we have that

$$
\boldsymbol{\alpha}(\mathbf{x}, \mathbf{y})=\mathbf{0} \quad \text { if } \quad|\mathbf{y}-\mathbf{x}| \geq \varepsilon
$$

and similarly for $\boldsymbol{\beta}$ and $\boldsymbol{\gamma}$; here, $\varepsilon>0$ denotes a cut-off or horizon parameter which is not necessarily small and which defines the extent of interactions. We then have that

$$
\Omega_{c}=\left\{\mathbf{y} \in \mathbb{R}^{n} \backslash \Omega_{s}:|\mathbf{y}-\mathbf{x}|<\varepsilon \quad \text { for } \mathbf{x} \in \Omega_{s}\right\} .
$$

We also define the domain

$\Omega_{-}:=\left\{\mathbf{y} \in \Omega_{s}:|\mathbf{y}-\mathbf{x}|<\varepsilon\right.$ for $\left.\mathbf{x} \in \mathbb{R}^{n} \backslash \Omega_{s}\right\}=\left\{\mathbf{y} \in \Omega_{s}:|\mathbf{y}-\mathbf{x}|<\varepsilon\right.$ for $\left.\mathbf{x} \in \Omega_{c}\right\}$,

where the last equality follows from (4.10). See Figure 2 for an illustration.

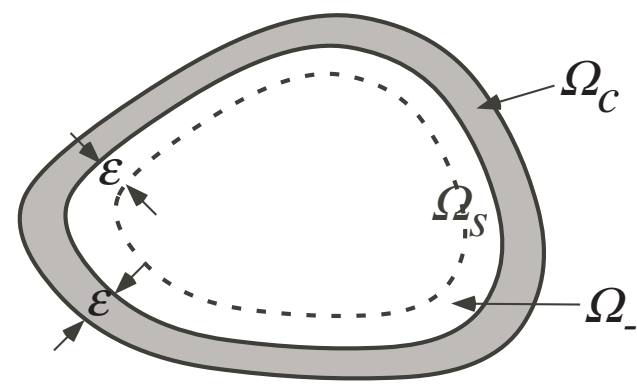

Fig. 2. For a localized kernel, the domain $\Omega_{s}$ and the interaction regions $\Omega_{c}$ and $\Omega_{-}$whose thicknesses are given by the horizon $\varepsilon$.

From (4.2a) for the first and third equalities and (4.10) for the second, we have that

$$
\begin{aligned}
\int_{\Omega_{s}} \mathcal{N}(\boldsymbol{\nu}) d \mathbf{x} & =\int_{\Omega_{s}} \int_{\Omega_{c}}\left(\boldsymbol{\nu}+\boldsymbol{\nu}^{\prime}\right) \cdot \boldsymbol{\alpha} d \mathbf{y} d \mathbf{x} \\
& =\int_{\Omega_{-}} \int_{\Omega_{c}}\left(\boldsymbol{\nu}+\boldsymbol{\nu}^{\prime}\right) \cdot \boldsymbol{\alpha} d \mathbf{y} d \mathbf{x}=\int_{\Omega_{-}} \mathcal{N}(\boldsymbol{\nu}) d \mathbf{x} .
\end{aligned}
$$

The first term is the nonlocal flux from $\Omega_{s}$ into $\Omega_{c}$; according to the last term, only points in $\Omega_{-} \subset \Omega_{s}$ contribute to that flux. Similar observations can be made for the operators $\mathcal{S}$ and $\mathcal{T}$.

Of course, all the definitions and results of Sections 3.1-4.3 hold for the case of localized kernels. 


\section{Relations between nonlocal and differential operators}

In this section, we choose particular kernel functions $\boldsymbol{\alpha}, \boldsymbol{\beta}$, and $\boldsymbol{\gamma}$ that lead to identifications of the nonlocal operators with their differential counterparts. In Section 5.1, the identification is made in a distributional sense whereas, in Section 5.2, we demonstrate circumstances under which the nonlocal point operators are weak representations of the divergence, gradient, and curl differential operators. For the sake of brevity, we mostly consider the nonlocal point divergence operator $\mathcal{D}$ and its adjoint operator $\mathcal{D}^{*}$. However, analogous results also hold for the nonlocal gradient and curl operators $\mathcal{G}$ and $\mathcal{C}$, respectively, and their adjoints. We then consider, in Section 5.3, a connection between the nonlocal and local Gauss theorems made with the help of two remarkable results given in Ref. 14 .

Throughout this section, we set $k=n$ in Definition 3.1, Theorem 3.1, and all subsequent results.

\subsection{Identification of nonlocal operators with differential operators in a distributional sense}

We begin with the identification of the nonlocal point divergence operator $\mathcal{D}$ with the divergence differential operator $\nabla \cdot$. This identification is subject to the understanding that the former operates on two-point functions whereas the latter operates on point functions. Where there might be ambiguity in applying differential operators to two-point function, we identify explicitly the variable of differentiation, e.g., $\nabla_{\mathbf{y}} \cdot \boldsymbol{\nu}(\mathbf{x}, \mathbf{y})$ denotes the divergence of $\boldsymbol{\nu}(\mathbf{x}, \mathbf{y})$ with respect to $\mathbf{y}$.

We consider special cases of localized kernels (see Section (4.4.3)) for which $\varepsilon \rightarrow$ 0 . As such, the "proofs" provided in this subsection are purely formal; they could be made rigorous through limiting processes involving sequences of $\varepsilon$-dependent kernels with $\varepsilon \rightarrow 0$.

We first relate, in a distributional sense, the nonlocal divergence with the local differential divergence.

Proposition 5.1. Let $\boldsymbol{\nu} \in \mathcal{C}_{0}^{\infty}\left(\mathbb{R}^{n} \times \mathbb{R}^{n}\right)$, i.e., $\boldsymbol{\nu}$ belongs to the space of compactly supported infinitely differentiable functions. Select the (antisymmetric) distribution

$$
\boldsymbol{\alpha}(\mathbf{y}, \mathbf{x})=-\nabla_{\mathbf{y}} \delta(\mathbf{y}-\mathbf{x})
$$

where $\nabla_{\mathbf{y}}$ and $\delta(\mathbf{y}-\mathbf{x})$ denote the differential gradient with respect to $\mathbf{y}$ and the Dirac delta distribution, respectively. Then,

$$
\mathcal{D}(\boldsymbol{\nu})(\mathbf{x})=\nabla \cdot \boldsymbol{\nu}(\mathbf{x}, \mathbf{x}) \quad \forall \mathbf{x} \in \mathbb{R}^{n}
$$

where $\nabla \cdot$ denotes the differential divergence operator. 
Proof. We have, by the chain rule and for $\boldsymbol{\alpha}$ given by (5.1),

$$
\begin{aligned}
\nabla \cdot \boldsymbol{\nu}(\mathbf{x}, \mathbf{x}) & =\left.\left(\nabla_{\mathbf{x}} \cdot \boldsymbol{\nu}(\mathbf{x}, \mathbf{y})\right)\right|_{\mathbf{y}=\mathbf{x}}+\left.\left(\nabla_{\mathbf{y}} \cdot \boldsymbol{\nu}(\mathbf{x}, \mathbf{y})\right)\right|_{\mathbf{y}=\mathbf{x}} \\
& =\left.\left(\nabla_{\mathbf{y}} \cdot \boldsymbol{\nu}(\mathbf{y}, \mathbf{x})\right)\right|_{\mathbf{y}=\mathbf{x}}+\left.\left(\nabla_{\mathbf{y}} \cdot \boldsymbol{\nu}(\mathbf{x}, \mathbf{y})\right)\right|_{\mathbf{y}=\mathbf{x}} \\
& =\left.\left(\nabla_{\mathbf{y}} \cdot(\boldsymbol{\nu}(\mathbf{y}, \mathbf{x})+\boldsymbol{\nu}(\mathbf{x}, \mathbf{y}))\right)\right|_{\mathbf{y}=\mathbf{x}} \\
& =\int_{\mathbb{R}^{n}} \nabla_{\mathbf{y}} \cdot(\boldsymbol{\nu}(\mathbf{y}, \mathbf{x})+\boldsymbol{\nu}(\mathbf{x}, \mathbf{y})) \delta(\mathbf{y}-\mathbf{x}) d \mathbf{y} \\
& =-\int_{\mathbb{R}^{n}}(\boldsymbol{\nu}(\mathbf{y}, \mathbf{x})+\boldsymbol{\nu}(\mathbf{x}, \mathbf{y})) \cdot \nabla_{\mathbf{y}} \delta(\mathbf{y}-\mathbf{x}) d \mathbf{y} \\
& =\int_{\mathbb{R}^{n}}(\boldsymbol{\nu}(\mathbf{y}, \mathbf{x})+\boldsymbol{\nu}(\mathbf{x}, \mathbf{y})) \cdot \boldsymbol{\alpha}(\mathbf{x}, \mathbf{y}) d \mathbf{y}=\mathcal{D}(\boldsymbol{\nu})(\mathbf{x}),
\end{aligned}
$$

where the last equality follows from (3.1a).

The following proposition shows that the average of the action of $\mathcal{D}^{*}$ on a point function can be related, in a distributional sense, to the action of $-\nabla$ on that function.

Proposition 5.2. Let $u \in \mathcal{C}_{0}^{\infty}(\Omega)$ and select $\boldsymbol{\alpha}$ as in (5.1). Then,

$$
\int_{\mathbb{R}^{n}} \mathcal{D}^{*}(u) d \mathbf{y}=-\nabla u \quad \forall \mathbf{x} \in \mathbb{R}^{n} .
$$

Proof. Starting with (3.4a) and (5.1), we have that

$$
\begin{aligned}
\int_{\mathbb{R}^{n}} \mathcal{D}^{*}(u) d \mathbf{y} & =\int_{\mathbb{R}^{n}}(u(\mathbf{y})-u(\mathbf{x})) \nabla_{\mathbf{y}} \delta(\mathbf{y}-\mathbf{x}) d \mathbf{y} \\
& =-\int_{\mathbb{R}^{n}} \delta(\mathbf{y}-\mathbf{x}) \nabla_{\mathbf{y}}(u(\mathbf{y})-u(\mathbf{x})) d \mathbf{y} \\
& =-\int_{\mathbb{R}^{n}} \delta(\mathbf{y}-\mathbf{x}) \nabla_{\mathbf{y}} u(\mathbf{y}) d \mathbf{y}=-\nabla u(\mathbf{x})=(\nabla \cdot)^{*} u(\mathbf{x}) .
\end{aligned}
$$

We next relate, in a distributional sense, the interaction operator $\mathcal{N}$ with the normal component along the boundary.

Proposition 5.3. Assume the hypotheses of Proposition 5.1. Then, for all smooth functions $u(\mathbf{x}): \Omega_{s} \rightarrow \mathbb{R}^{n}$,

$$
\int_{\Omega_{s}} u \mathcal{N}(\boldsymbol{\nu}) d \mathbf{x}=\int_{\partial \Omega_{s}} u(\mathbf{x}) \boldsymbol{\nu}(\mathbf{x}, \mathbf{x}) \cdot \mathbf{n} d A,
$$

where $\mathbf{n}$ denotes the unit outward-pointing normal vector along $\partial \Omega_{s}$. Thus, $\mathcal{N}(\boldsymbol{\nu})$ is a delta measure concentrated on $\partial \Omega_{s}$ with coefficient (weight) $\boldsymbol{\nu}(\mathbf{x}, \mathbf{x}) \cdot \mathbf{n}$.

Proof. With $\boldsymbol{\alpha}$ chosen as in (5.1) and using the definition (3.4) for the operator 
$\mathcal{D}^{*}$, we have that

$$
\begin{aligned}
-\int_{\Omega_{s}} \int_{\Omega_{s}} \mathcal{D}^{*}(u) \cdot \boldsymbol{\nu} d \mathbf{y} d \mathbf{x}= & \int_{\Omega_{s}} \int_{\Omega_{s}}(u(\mathbf{y})-u(\mathbf{x})) \boldsymbol{\nu}(\mathbf{x}, \mathbf{y}) \cdot \boldsymbol{\alpha}(\mathbf{x}, \mathbf{y}) d \mathbf{y} d \mathbf{x} \\
= & -\int_{\Omega_{s}} \int_{\Omega_{s}}(u(\mathbf{y})-u(\mathbf{x})) \boldsymbol{\nu}(\mathbf{x}, \mathbf{y}) \cdot \nabla_{\mathbf{y}} \delta(\mathbf{y}-\mathbf{x}) d \mathbf{y} d \mathbf{x} \\
= & \int_{\Omega_{s}} \int_{\Omega_{s}} \nabla_{\mathbf{y}}(u(\mathbf{y})-u(\mathbf{x})) \cdot \boldsymbol{\nu}(\mathbf{x}, \mathbf{y}) \delta(\mathbf{y}-\mathbf{x}) d \mathbf{y} d \mathbf{x} \\
& \quad+\int_{\Omega_{s}} \int_{\Omega_{s}}(u(\mathbf{y})-u(\mathbf{x})) \delta(\mathbf{y}-\mathbf{x}) \nabla_{\mathbf{y}} \cdot \boldsymbol{\nu}(\mathbf{x}, \mathbf{y}) d \mathbf{y} d \mathbf{x} \\
& -\int_{\Omega_{s}} \int_{\partial \Omega_{s}}(u(\mathbf{y})-u(\mathbf{x})) \delta(\mathbf{y}-\mathbf{x}) \boldsymbol{\nu}(\mathbf{x}, \mathbf{y}) \cdot \mathbf{n} d \mathbf{y} d \mathbf{x} \\
= & \int_{\Omega_{s}} \int_{\Omega_{s}} \nabla_{\mathbf{y}} u(\mathbf{y}) \cdot \boldsymbol{\nu}(\mathbf{x}, \mathbf{y}) \delta(\mathbf{y}-\mathbf{x}) d \mathbf{y} d \mathbf{x} \\
= & \int_{\Omega_{s}} \nabla u(\mathbf{x}) \cdot \boldsymbol{\nu}(\mathbf{x}, \mathbf{x}) d \mathbf{x},
\end{aligned}
$$

where the third equality follows because $u(\mathbf{y})-u(\mathbf{x})=0$ whenever $\mathbf{y}=\mathbf{x}$ and the fourth equality follows because $\nabla_{\mathbf{y}} u(\mathbf{x})=0$. Also, with $\boldsymbol{\alpha}$ chosen as in (5.1) and using (5.2), we have that

$\int_{\Omega_{s}} u \mathcal{D}(\boldsymbol{\nu}) d \mathbf{x}=\int_{\Omega_{s}} u(\mathbf{x}) \nabla \cdot \boldsymbol{\nu}(\mathbf{x}, \mathbf{x}) d \mathbf{x}=\int_{\partial \Omega_{s}} u(\mathbf{x}) \boldsymbol{\nu}(\mathbf{x}, \mathbf{x}) \cdot \mathbf{n} d A-\int_{\Omega_{s}} \nabla u(\mathbf{x}) \cdot \boldsymbol{\nu}(\mathbf{x}, \mathbf{x}) d \mathbf{x}$

Substituting the last two results into (4.5) results in

$$
\int_{\Omega_{s}} u \mathcal{N}(\boldsymbol{\nu}) d \mathbf{x}=\int_{\Omega_{s}} u \mathcal{D}(\boldsymbol{\nu}) d \mathbf{x}-\int_{\Omega_{s}} \int_{\Omega_{s}} \mathcal{D}^{*}(u) \cdot \boldsymbol{\nu} d \mathbf{y} d \mathbf{x}=\int_{\partial \Omega_{s}} u(\mathbf{x}) \boldsymbol{\nu}(\mathbf{x}, \mathbf{x}) \cdot \mathbf{n} d A_{\bullet}
$$

Now consider the composition of the nonlocal divergence operator and its adjoint, i.e., $\mathcal{D}\left(\mathcal{D}^{*}\right)$, which, according to the next proposition, can be identified, in the sense of distributions, with $-\Delta=\nabla \cdot(-\nabla)$, where $\Delta$ denotes the Laplace operator

Proposition 5.4. Let $u \in \mathcal{C}_{0}^{\infty}\left(\mathbb{R}^{n}\right)$ and select $|\boldsymbol{\alpha}(\mathbf{x}, \mathbf{y})|^{2}=\frac{1}{2} \Delta_{\mathbf{y}} \delta(\mathbf{y}-\mathbf{x})$. Then,

$$
\mathcal{D}\left(\mathcal{D}^{*} u(\mathbf{x})\right)=-\Delta u(\mathbf{x}) \quad \forall \mathbf{x} \in \mathbb{R}^{n}
$$


Proof. From (3.1a) and (3.4a) we have

$$
\begin{aligned}
\mathcal{D}\left(\mathcal{D}^{*} u\right)(\mathbf{x}) & =-2 \int_{\mathbb{R}^{n}}(u(\mathbf{y})-u(\mathbf{x}))|\boldsymbol{\alpha}(\mathbf{x})|^{2} d \mathbf{y} \\
& =-\int_{\mathbb{R}^{n}}(u(\mathbf{y})-u(\mathbf{x})) \Delta_{\mathbf{y}} \delta(\mathbf{y}-\mathbf{x}) d \mathbf{y} \\
& =-\int_{\mathbb{R}^{n}} \delta(\mathbf{y}-\mathbf{x}) \Delta_{\mathbf{y}}(u(\mathbf{y})-u(\mathbf{x})) d \mathbf{y} \\
& =-\int_{\mathbb{R}^{n}} \delta(\mathbf{y}-\mathbf{x}) \Delta_{\mathbf{y}} u(\mathbf{y}) d \mathbf{y} \\
& =-\Delta u(\mathbf{x})=-\nabla \cdot \nabla u(\mathbf{x})=\nabla \cdot(\nabla \cdot)^{*} u(\mathbf{x}),
\end{aligned}
$$

where the differential Green's second identity is used for the third equality.

\subsection{Relations between weighted nonlocal operators and weak representations of differential operators}

The classical differential calculus only involves operators mapping point functions to point functions; on the other hand, the nonlocal operators defined earlier map two-point functions to point functions or, in case of the nonlocal adjoint operators, point functions to two-point functions. To further demonstrate that the nonlocal operators correspond to nonlocal versions of the classical divergence, gradient, and curl differential operators, we use the nonlocal operators $\mathcal{D}, \mathcal{G}$, and $\mathcal{C}$ to define, in Section 5.2.1, corresponding nonlocal weighted operators that map point functions to point functions. We also show that the adjoint operators corresponding to the weighted operators are weighted integrals of the nonlocal adjoint operators $\mathcal{D}^{*}, \mathcal{G}^{*}$, and $\mathcal{C}^{*}$. Then, in Section 5.2.2, the weighted operators are rigorously shown to be nonlocal versions of the corresponding differential operators.

\subsubsection{Nonlocal weighted operators}

The nonlocal point operators defined in (3.1) induce new operators, referred to as weighted operators. Definition 5.1 and 5.2 and Proposition 5.5 hold for general $k$ and $m$ although, when we apply them in Section 5.2 .2 , we set $k=m=n$.

Definition 5.1. Let $\omega(\mathbf{x}, \mathbf{y}): \mathbb{R}^{n} \times \mathbb{R}^{n} \rightarrow \mathbb{R}$ denote a non-negative scalar-valued two-point function. Let the operators $\mathcal{D}, \mathcal{G}$, and $\mathcal{C}$ be defined as in (3.1). Then, given the point function $\mathbf{u}(\mathbf{x}): \mathbb{R}^{n} \rightarrow \mathbb{R}^{k}$, the weighted nonlocal divergence operator $\mathcal{D}_{\omega}(\mathbf{u}): \mathbb{R}^{n} \rightarrow \mathbb{R}$ is defined by its action on $\mathbf{u}$ by

$$
\mathcal{D}_{\omega}(\mathbf{u})(\mathbf{x}):=\mathcal{D}(\omega(\mathbf{x}, \mathbf{y}) \mathbf{u}(\mathbf{x})) \quad \text { for } \mathbf{x} \in \mathbb{R}^{n} .
$$

Given the point function $u(\mathbf{x}): \mathbb{R}^{n} \rightarrow \mathbb{R}$, the weighted nonlocal gradient operator $\mathcal{G}_{\omega}(u): \mathbb{R}^{n} \rightarrow \mathbb{R}^{k}$ is defined by its action on $u$ by

$$
\mathcal{G}_{\omega}(u)(\mathbf{x}):=\mathcal{G}(\omega(\mathbf{x}, \mathbf{y}) u(\mathbf{x})) \quad \text { for } \mathbf{x} \in \mathbb{R}^{n} .
$$


Given the point function $\mathbf{w}(\mathbf{x}): \mathbb{R}^{n} \rightarrow \mathbb{R}^{3}$, the weighted nonlocal curl operator $\mathcal{C}_{\omega}(\mathbf{u}): \mathbb{R}^{n} \rightarrow \mathbb{R}^{3}$ is defined by its action on $\mathbf{w}$ by

$$
\mathcal{C}_{\omega}(\mathbf{w})(\mathbf{x}):=\mathcal{C}(\omega(\mathbf{x}, \mathbf{y}) \mathbf{w}(\mathbf{x})) \quad \text { for } \mathbf{x} \in \mathbb{R}^{n} .
$$

The following result shows that the adjoint operators corresponding to the weighted nonlocal operators are determined as weighted integrals of the corresponding nonlocal two-point adjoint operators (3.4).

Proposition 5.5. Let $\omega(\mathbf{x}, \mathbf{y}): \mathbb{R}^{n} \times \mathbb{R}^{n} \rightarrow \mathbb{R}$ denote a non-negative scalar twopoint function and let $\mathcal{D}^{*}, \mathcal{G}^{*}$, and $\mathcal{C}^{*}$ denote the nonlocal adjoint operators given in (3.4). The action on $u$ of the adjoint operator $\mathcal{D}_{\omega}^{*}(u)(\mathbf{x}): \mathbb{R}^{n} \rightarrow \mathbb{R}^{k}$ corresponding to the weighted nonlocal divergence operator $\mathcal{D}_{\omega}$ is given by

$$
\mathcal{D}_{\omega}^{*}(u)(\mathbf{x})=\int_{\mathbb{R}^{n}} \mathcal{D}^{*}(u)(\mathbf{x}, \mathbf{y}) \omega(\mathbf{x}, \mathbf{y}) d \mathbf{y} \quad \text { for } \mathbf{x} \in \mathbb{R}^{n}
$$

for scalar point functions $u(\mathbf{x}): \mathbb{R}^{n} \rightarrow \mathbb{R}$. The action on $\mathbf{u}$ of the adjoint operator $\mathcal{G}_{\omega}^{*}(\mathbf{v})(\mathbf{x}): \mathbb{R}^{n} \rightarrow \mathbb{R}$ corresponding to the weighted nonlocal gradient operator $\mathcal{G}_{\omega}$ is given by

$$
\mathcal{G}_{\omega}^{*}(\mathbf{v})(\mathbf{x})=\int_{\mathbb{R}^{n}} \mathcal{G}^{*}(\mathbf{v})(\mathbf{x}, \mathbf{y}) \omega(\mathbf{x}, \mathbf{y}) d \mathbf{y} \quad \text { for } \mathbf{x} \in \mathbb{R}^{n} .
$$

for vector point functions $\mathbf{v}(\mathbf{x}): \mathbb{R}^{n} \rightarrow \mathbb{R}^{k}$. The action on $\mathbf{w}$ of the adjoint operator $\mathcal{C}_{\omega}^{*}(\mathbf{w})(\mathbf{x}): \mathbb{R}^{n} \rightarrow \mathbb{R}^{3}$ corresponding to the weighted nonlocal curl operator $\mathcal{C}_{\omega}$ is given by

$$
\mathcal{C}_{\omega}^{*}(\mathbf{w})(\mathbf{x})=\int_{\mathbb{R}^{n}} \mathcal{C}^{*}(\mathbf{w})(\mathbf{x}, \mathbf{y}) \omega(\mathbf{x}, \mathbf{y}) d \mathbf{y} \quad \text { for } \mathbf{x} \in \mathbb{R}^{n}
$$

for vector point functions $\mathbf{w}(\mathbf{x}): \mathbb{R}^{n} \rightarrow \mathbb{R}^{3}$.

Proof. We have that

$$
\begin{aligned}
\left(\mathcal{D}_{\omega}(\mathbf{u}), u\right)_{\mathbb{R}^{n}} & =\int_{\mathbb{R}^{n}} u(\mathbf{x}) \mathcal{D}(\omega(\mathbf{x}, \mathbf{y}) \mathbf{u}(\mathbf{x})) d \mathbf{x} \\
& =\int_{\mathbb{R}^{n}} \int_{\mathbb{R}^{n}} \mathcal{D}^{*}(u)(\mathbf{x}, \mathbf{y}) \cdot(\omega(\mathbf{x}, \mathbf{y}) \mathbf{u}(\mathbf{x})) d \mathbf{y} d \mathbf{x} \\
& =\int_{\mathbb{R}^{n}}\left(\int_{\mathbb{R}^{n}} \omega(\mathbf{x}, \mathbf{y}) \mathcal{D}^{*}(u)(\mathbf{x}, \mathbf{y}) d \mathbf{y}\right) \cdot \mathbf{u}(\mathbf{x}) d \mathbf{x}
\end{aligned}
$$

where (5.4a) is used for the first equality and (3.3) and (3.4a) for the second. But, by definition, the adjoint operator $\mathcal{D}_{\omega}^{*}(\cdot)$ corresponding to the operator $\mathcal{D}_{\omega}(\cdot)$ satisfies

$$
\left(u, \mathcal{D}_{\omega}(\mathbf{u})\right)_{\mathbb{R}^{n}}=\left(\mathcal{D}_{\omega}^{*}(u), \mathbf{u}\right)_{\mathbb{R}^{n}} .
$$

Comparing the last two results yields (5.4a). The conclusions (5.4b) and (5.4c) are derived in a similar fashion.

The definition given in (5.4a) and the result (5.5a) can be extended to tensors and vectors, respectively. 
Definition 5.2. Let $\omega(\mathbf{x}, \mathbf{y}): \mathbb{R}^{n} \times \mathbb{R}^{n} \rightarrow \mathbb{R}$ denote a non-negative scalar twopoint function and let $\mathcal{D}_{t}$ be given as in $(3.8 \mathrm{~b})$. Given the tensor point function $\mathbf{U}(\mathbf{x}): \mathbb{R}^{n} \rightarrow \mathbb{R}^{\ell \times k}$, the weighted nonlocal divergence operator $\mathcal{D}_{t, \omega}(\mathbf{U}): \mathbb{R}^{n} \rightarrow \mathbb{R}^{\ell}$ for tensors is defined by

$$
\mathcal{D}_{t, \omega}(\mathbf{U})(\mathbf{x}):=\mathcal{D}_{t}(\omega(\mathbf{x}, \mathbf{y}) \mathbf{U}(\mathbf{x}))(\mathbf{x}) \text { for } \mathbf{x} \in \mathbb{R}^{n} .
$$

Corollary 5.1. The adjoint operator $\mathcal{D}_{t, \omega}^{*}(\mathbf{u})(\mathbf{x}): \mathbb{R}^{n} \rightarrow \mathbb{R}^{\ell \times k}$ corresponding to the operator $\mathcal{D}_{t, \omega}$ is given by

$$
\mathcal{D}_{t, \omega}^{*}(\mathbf{u})(\mathbf{x})=\int_{\mathbb{R}^{n}} \mathcal{D}_{t}^{*}(\mathbf{u})(\mathbf{x}, \mathbf{y}) \omega(\mathbf{x}, \mathbf{y}) d \mathbf{y} \quad \text { for } \mathbf{x} \in \mathbb{R}^{n}
$$

for vector point functions $\mathbf{u}: \mathbb{R}^{n} \rightarrow \mathbb{R}^{\ell}$.

Similar results hold for the operator $\mathcal{G}_{v}$.

\subsubsection{Relationships between weighted operators and differential operators}

We have seen that the nonlocal operators satisfy many properties that mimic their differential counterparts. In particular, we established, in Section 5.1, that the nonlocal point operators can be identified with the corresponding differential operators in a distributional sense. Here, we establish rigorous connections between the weighted operators and their differential counterparts for the case of special localized kernels (see Section 4.10), i.e., we introduce the horizon parameter $\varepsilon>0$ and analyze what occurs as $\varepsilon \rightarrow 0$, that is, in the local limit. To this end, we define

$$
B_{\varepsilon}(\mathbf{x}):=\left\{\mathbf{y} \in \mathbb{R}^{n}:|\mathbf{y}-\mathbf{x}|<\varepsilon\right\}
$$

and let $\phi$ denote a positive radial function satisfying a normalization condition

$$
\int_{B_{\varepsilon}(\mathbf{x})}|\mathbf{y}-\mathbf{x}|^{2} \phi(|\mathbf{y}-\mathbf{x}|) d \mathbf{y}=n
$$

where $n$ denotes the space dimension. We then choose

$$
\begin{cases}\boldsymbol{\alpha}(\mathbf{x}, \mathbf{y})=\frac{\mathbf{y}-\mathbf{x}}{|\mathbf{y}-\mathbf{x}|} & \text { for } \mathbf{x} \neq \mathbf{y} \\ \omega(\mathbf{x}, \mathbf{y})= \begin{cases}|\mathbf{y}-\mathbf{x}| \phi(|\mathbf{y}-\mathbf{x}|) & \mathbf{y} \in B_{\varepsilon}(\mathbf{x}) \\ 0 & \text { otherwise. }\end{cases} \end{cases}
$$

Note that $\boldsymbol{\alpha}$ is an antisymmetric function whereas $\omega$ is a symmetric function. We then have, for a scalar function $u$, that the components of $\mathcal{G}_{\omega}(u)$ and $\mathcal{D}_{\omega}^{*}(u)$ given by $(5.4 \mathrm{~b})$ and $(5.5 \mathrm{a})$, respectively, are given by, for $j=1, \ldots, n$,

$$
\begin{aligned}
& \mathrm{d}_{j} u(\mathbf{x}):=\int_{B_{\varepsilon}(\mathbf{0})}(u(\mathbf{x}+\mathbf{z})+u(\mathbf{x})) z_{j} \phi(|\mathbf{z}|) d \mathbf{z} \\
& \mathrm{d}_{j}^{*} u(\mathbf{x}):=-\int_{B_{\varepsilon}(\mathbf{0})}(u(\mathbf{x}+\mathbf{z})-u(\mathbf{x})) z_{j} \phi(|\mathbf{z}|) d \mathbf{z},
\end{aligned}
$$


where $z_{j}$ denotes the $j$-th component of $\mathbf{z}$.

The following result ensues.

Lemma 5.1. Assume that $\omega$ and $\boldsymbol{\alpha}$ are defined as in (5.9). Let $u: \mathbb{R}^{n} \rightarrow \mathbb{R}$ and let $\mathrm{d}_{j} u(\mathbf{x})$ and $\mathrm{d}_{j}^{*} u(\mathbf{x})$ denote the $j$-th components of $\mathcal{G}_{\omega}(u)$ and $\mathcal{D}_{\omega}^{*}(u)$, respectively. Then,

$$
\mathrm{d}_{j} u=-\mathrm{d}_{j}^{*} u .
$$

Proof. From (5.10) we have

$$
\mathrm{d}_{j} u(\mathbf{x})+\mathrm{d}_{j}^{*} u(\mathbf{x})=-2 u(\mathbf{x}) \int_{B_{\varepsilon}(\mathbf{0})} y_{j} \phi(|\mathbf{y}|) d \mathbf{y}=0
$$

from which the result directly follows.

Based on this lemma and the definition of the weighted operators, we have the following results.

Corollary 5.2. Under the same conditions as in Lemma 5.1, we have

$$
\mathcal{D}_{\omega}=-\mathcal{G}_{\omega}^{*}, \quad \mathcal{G}_{\omega}=-\mathcal{D}_{\omega}^{*}, \quad \text { and } \quad \mathcal{C}_{\omega}=\mathcal{C}_{\omega}^{*} .
$$

Thus, under the hypotheses of this corollary, the identities in (5.12) mimic their counterparts in the vector calculus for differential operators. Identities such as those in Proposition 3.1 do not generally hold for the weighted operators due to their nonlocal properties. ${ }^{\mathrm{p}}$

Synergistic with the results of Section 5.1, we have the following.

Corollary 5.3. Select the singular distribution

$$
\mathbf{z}_{j} \phi(|\mathbf{z}|)=-\partial_{j} \delta(\mathbf{z}),
$$

where $\partial_{j} u$ denotes the weak derivative of $u$ with respect to $x_{j}$. Then,

$$
\mathrm{d}_{j}=\partial_{j} \quad \text { and } \quad \mathrm{d}_{j}^{*}=-\partial_{j} .
$$

The following proposition demonstrates that the components of the weighted gradient operator $\mathcal{G}_{\omega}$ or, by $(5.12), \mathcal{D}_{\omega}^{*}$, converge, as $\varepsilon \rightarrow 0$, to the corresponding spatial derivatives.

Proposition 5.6. Let $\omega$ be defined as in (5.9) with $\phi$ satisfying (5.8). Then, for $j=1, \ldots, n$, the weighted operators $\mathrm{d}_{j}$ and $\mathrm{d}_{j}^{*}$ defined by (5.10) are bounded linear operators from $H^{1}\left(\mathbb{R}^{n}\right)$ to $L^{2}\left(\mathbb{R}^{n}\right)$. Moreover, if $u \in H^{1}\left(\mathbb{R}^{n}\right)$, then as $\varepsilon \rightarrow 0$

$$
\begin{aligned}
\left\|\mathbf{d}_{j} u-\partial_{j} u\right\|_{L^{2}\left(\mathbb{R}^{n}\right)} & \rightarrow 0 \\
\left\|\mathbf{d}_{j}^{*} u+\partial_{j} u\right\|_{L^{2}\left(\mathbb{R}^{n}\right)} & \rightarrow 0,
\end{aligned}
$$

PThe identities in Lemma 5.1 and Corollary 5.2 also hold for more general choices of $\boldsymbol{\alpha}$ and $\omega$, namely, those such that $\boldsymbol{\alpha}$ is antisymmetric and $\omega$ is a radial function with support in $B_{\varepsilon}(\mathbf{x})$. 
where $\partial_{j} u$ denotes the weak partial derivative of $u$ with respect to $x_{j}$. Moreover, if

$$
\int_{B_{\varepsilon}(0)}|\mathbf{z}|^{1+s} \phi(|\mathbf{z}|) d \mathbf{z}<\infty \quad \text { for some } 0 \leq s \leq 1,
$$

then, for $j=1, \ldots, n$, the weighted operators $\mathrm{d}_{j}$ and $\mathrm{d}_{j}^{*}$ are bounded linear operators from $H^{t}\left(\mathbb{R}^{n}\right)$ to $H^{t-s}\left(\mathbb{R}^{n}\right)$ for any $t \geq 0$.

Proof. The conclusion (5.13a) is equivalent to $\left\|\widehat{\mathrm{d}_{j} u}-\widehat{\partial_{j} u}\right\|_{L^{2}\left(\mathbb{R}^{n}\right)} \rightarrow 0$, where $\widehat{\mathrm{d}_{j} u}$ and $\widehat{\partial_{j} u}$ denote the Fourier transforms of $\mathrm{d}_{j} u$ and $\partial_{j} u$, respectively.

Under the condition (5.13c) for $s \in[0,1]$, if $u \in H^{t}\left(\mathbb{R}^{n}\right)$ for $t \geq 0$, then

$$
\begin{aligned}
\widehat{\mathrm{d}_{j} u}= & \int_{B_{\varepsilon}(\mathbf{0})}\left(e^{i \mathbf{y} \cdot \boldsymbol{\xi}}+1\right) \widehat{u}(\boldsymbol{\xi}) y_{j} \phi(|\mathbf{y}|) d \mathbf{y} \\
= & i \int_{B_{\varepsilon}(\mathbf{0})} \sin (\mathbf{y} \cdot \boldsymbol{\xi}) \widehat{u}(\boldsymbol{\xi}) y_{j} \phi(|\mathbf{y}|) d \mathbf{y} \\
= & i \int_{B_{\varepsilon}(\mathbf{0})}\left(\sin \left(y_{j} \xi_{j}\right) \cos \left(\sum_{k \neq j} y_{k} \xi_{k}\right)\right. \\
& \left.+\cos \left(y_{j} \xi_{j}\right) \sin \left(\sum_{k \neq j} y_{k} \xi_{k}\right)\right) \widehat{u}(\boldsymbol{\xi}) y_{j} \phi(|\mathbf{y}|) d \mathbf{y} \\
= & i \widehat{u}(\boldsymbol{\xi}) \int_{B_{\varepsilon}(\mathbf{0})} \sin \left(y_{j} \xi_{j}\right) \cos \left(\sum_{k \neq j} y_{i} \xi_{k}\right) y_{j} \phi(|\mathbf{y}|) d \mathbf{y},
\end{aligned}
$$

where the second and fourth equalities hold because of the symmetry of the domain of integration and the antisymmetry of the integrand with respect to the integration variable, respectively. Because for any $0 \leq s \leq 1,\left|\sin \left(y_{j} \xi_{j}\right) \cos \left(\sum_{k \neq j} y_{i} \xi_{k}\right)\right| \leq$ $\left|y_{j} \xi_{j}\right|^{s}$, we have

$$
\left|\widehat{\mathbf{d}_{j} u}\right| \leq\left|\xi^{s} \widehat{u}(\boldsymbol{\xi})\right| \int_{B_{\varepsilon}(\mathbf{0})}\left|y_{j}\right|^{1+s} \phi(|\mathbf{y}|) d \mathbf{y}
$$

so that $\widehat{\mathrm{d}_{j} u} \in H^{t-s}\left(\mathbb{R}^{n}\right)$. In particular, for $t=s=1$, we obtain that, under the condition (5.8), the weighted operators $\mathrm{d}_{j}, j=1, \ldots, n$, defined in (5.10a) are bounded linear operators from $H^{1}\left(\mathbb{R}^{n}\right)$ to $L^{2}\left(\mathbb{R}^{n}\right)$.

If $u \in H^{1}\left(\mathbb{R}^{n}\right)$, we have that

$$
\begin{aligned}
\left|\widehat{\mathrm{d}_{j} u}-\widehat{\partial_{j} u}\right| & \leq\left|\widehat{\mathrm{d}_{j} u}\right|+\left|\widehat{\partial_{j} u}\right| \\
& \leq \int_{B_{\varepsilon}(\mathbf{0})}\left|\sin \left(y_{j} \xi_{j}\right) \cos \left(\sum_{k \neq j} y_{k} \xi_{k}\right) \widehat{u}(\boldsymbol{\xi}) y_{j} \phi(|\mathbf{y}|)\right| d \mathbf{y}+\left|\xi_{j} \widehat{u}\right| \\
& \leq\left|\xi_{j} \widehat{u}\right| \int_{B_{\varepsilon}(\mathbf{0})} y_{j}^{2} \phi(|\mathbf{y}|) d \mathbf{y}+\left|\xi_{j} \widehat{u}\right| \\
& \leq 2\left|\xi_{j} \widehat{u}\right| \in L^{2}\left(\mathbb{R}^{n}\right),
\end{aligned}
$$


where the third inequality holds because $|\sin (x)| \leq|x|$ and $|\cos (x)| \leq 1$. By Taylor's theorem, we have

$$
\begin{aligned}
\widehat{\mathrm{d}_{j} u} & =i \int_{B_{\varepsilon}(\mathbf{0})}\left(y_{j} \xi_{j}+y_{j}^{3} \xi_{j}^{3} \cos \left(\theta_{1}\right) / 6\right)\left(1+\left(\sum_{k \neq j} y_{k} \xi_{k}\right)^{2} \cos \left(\theta_{2}\right) / 2\right) \widehat{u} y_{j} \phi(|\mathbf{y}|) d \mathbf{y} \\
& =i \xi_{j} \widehat{u} \int_{B_{\varepsilon}(\mathbf{0})} y_{j}^{2} \phi(|\mathbf{y}|) d \mathbf{y}+I_{\varepsilon}(\xi)=i \xi_{j} \widehat{u}+I_{\varepsilon}(\xi),
\end{aligned}
$$

where, for some $\theta_{1}$ and $\theta_{2}$,

$$
\begin{aligned}
I_{\varepsilon}(\xi):=\frac{i}{6} \int_{B_{\varepsilon}(\mathbf{0})} & y_{j}^{4} \cos \left(\theta_{1}\right) \phi(|\mathbf{y}|) d \mathbf{y} \xi_{j}^{3} \widehat{u} \\
+ & +\frac{i}{2} \int_{B_{\varepsilon}(\mathbf{0})} y_{j}^{2}\left(\sum_{k \neq j} y_{i} \xi_{i}\right)^{2} \cos \left(\theta_{2}\right) \phi(|\mathbf{y}|) d \mathbf{y} \xi_{j} \widehat{u} \\
& \quad+\frac{i}{12} \int_{B_{\varepsilon}(\mathbf{0})} y_{j}^{4}\left(\sum_{k \neq j} y_{k} \xi_{k}\right)^{2} \cos \left(\theta_{1}\right) \cos \left(\theta_{2}\right) \phi(|\mathbf{y}|) d \mathbf{y} \xi_{j}^{3} \widehat{u} .
\end{aligned}
$$

Because $\widehat{u}$ is bounded a.e., we see that, for any $\boldsymbol{\xi}$,

$$
\left|I_{\varepsilon}(\xi)\right| \leq \varepsilon^{2}\left|\xi_{j}^{3} \widehat{u}\right|+\varepsilon^{2}\left|\left(\sum_{k \neq j} \xi_{k}\right)^{2} \xi_{j} \widehat{u}\right|+\varepsilon^{4}\left|\left(\sum_{k \neq j} \xi_{k}\right)^{2} \xi_{j}^{3} \widehat{u}\right| \rightarrow 0 \quad \text { as } \quad \varepsilon \rightarrow 0 .
$$

Hence, we have

$$
\widehat{\mathrm{d}_{j} u} \rightarrow \widehat{\partial_{j} u} \quad \text { a.e. for } \boldsymbol{\xi} \in \mathbb{R}^{n} \quad \text { as } \varepsilon \rightarrow 0 .
$$

Then, by the dominated convergence theorem, we obtain

$$
\left\|\mathrm{d}_{j} u-\partial_{j} u\right\|_{L^{2}\left(\mathbb{R}^{n}\right)} \rightarrow 0 \text { as } \varepsilon \rightarrow 0 .
$$

Lemma 5.1 implies the same results hold for the operator $\mathrm{d}_{j}^{*}$.

The above lemma implies that if $\omega$ satisfies (5.13c) for $s=0$, then the weighted operators are bounded operators from $L^{2}\left(\mathbb{R}^{n}\right)$ to $L^{2}\left(\mathbb{R}^{n}\right)$. More generally, for $\phi$ satisfying (5.13c) with positive $s$, the operators $\mathrm{d}_{j}$ and $\mathrm{d}_{j}^{*}$ actually map a subspace of $L^{2}\left(\mathbb{R}^{n}\right)$, for instance the fractional Sobolev space $H^{s}\left(\mathbb{R}^{n}\right)$, to $L^{2}\left(\mathbb{R}^{n}\right)$, or even map $L^{2}\left(\mathbb{R}^{n}\right)$ to $H^{-s}\left(\mathbb{R}^{n}\right)$. We refer to Ref. 6 for related work.

A direct consequence of Lemma 5.6 is the following result.

Corollary 5.4. Under the condition of Lemma 5.6, the weighted operators $\mathcal{D}_{\omega}, \mathcal{G}_{\omega}$, and $\mathcal{C}_{\omega}$ and their adjoint operators $\mathcal{D}_{\omega}^{*}, \mathcal{G}_{\omega}^{*}$, and $\mathcal{C}_{\omega}^{*}$ are bounded linear operators from $H^{t}\left(\mathbb{R}^{n}\right)$ to $H^{t-s}\left(\mathbb{R}^{n}\right)$ for $0 \leq s \leq 1$, where $n=3$ for the weighted curl operators. Moreover, if $u \in H^{1}\left(\mathbb{R}^{n}\right)$ and $\mathbf{u} \in\left[H^{1}\left(\mathbb{R}^{n}\right)\right]^{n}$, then

$$
\begin{array}{lll}
\mathcal{D}_{\omega}(\mathbf{u}) \rightarrow \nabla \cdot \mathbf{u} & \mathcal{D}_{\omega}^{*}(u) \rightarrow-\nabla u \\
\mathcal{G}_{\omega}(u) \rightarrow \nabla u & & \mathcal{G}_{\omega}^{*}(\mathbf{u}) \rightarrow-\nabla \cdot \mathbf{u} \\
\mathcal{C}_{\omega}(\mathbf{u}) \rightarrow \nabla \times \mathbf{u} & & \mathcal{C}_{\omega}^{*}(\mathbf{u}) \rightarrow \nabla \times \mathbf{u},
\end{array}
$$


where the convergence as $\varepsilon \rightarrow 0$ is with respect to $L^{2}\left(\mathbb{R}^{n}\right)$.

Proof. We prove the convergence of the operator $\mathcal{D}_{\omega}(\mathbf{u})$ to the divergence differential operators. First, note that

$$
\mathcal{D}_{\omega}(\mathbf{u})=\sum_{i=1}^{n} \mathrm{~d}_{i} u_{i} \quad \text { and } \quad \nabla \cdot \mathbf{u}=\sum_{i=1}^{n} \partial_{i} u_{i}
$$

so that

$$
\left\|\mathcal{D}_{\omega}(\mathbf{u})-\nabla \cdot \mathbf{u}\right\|_{L^{2}\left(\mathbb{R}^{n}\right)} \leq \sum_{i=1}^{n}\left\|\mathrm{~d}_{i} u_{i}-\partial_{i} u_{i}\right\|_{L^{2}\left(\mathbb{R}^{n}\right)} \rightarrow 0 \quad \text { as } \varepsilon \rightarrow 0 .
$$

The remaining results can be proved in a similar fashion.

For the purpose of discussing nonlocal equations, we also need to consider combinations of the weighted operators such as $\mathcal{D}_{\omega}\left(\mathbf{C}_{1} \mathcal{D}_{\omega}^{*}(u)\right)$, where $\mathbf{C}_{1}(\mathbf{x})$ is a "constitutive" tensor point function that, for example, describes a point property of a material. Note that the two-point property is involved in the definition of the weighted operators. In the next corollary, we illustrate that whenever the horizon $\varepsilon$ goes to zero, the combinations of the weighted operators converge to their local counterparts.

Corollary 5.5. Let $u \in H^{1}\left(\mathbb{R}^{n}\right), \mathbf{u} \in\left[H^{1}\left(\mathbb{R}^{n}\right)\right]^{n}, \mathbf{C}_{1}: \mathbb{R}^{n} \rightarrow \mathbb{R}^{n} \times \mathbb{R}^{n}$ in $L^{\infty}\left(\mathbb{R}^{n} \times\right.$ $\left.\mathbb{R}^{n}\right)$, and $c_{2}: \mathbb{R}^{n} \rightarrow \mathbb{R}$ in $L^{\infty}\left(\mathbb{R}^{n}\right)$. Then,

$$
\begin{aligned}
& \mathcal{D}_{\omega}\left(\mathbf{C}_{1} \cdot \mathcal{D}_{\omega}^{*}(u)\right) \rightarrow-\nabla \cdot\left(\mathbf{C}_{1} \cdot \nabla u\right) \\
& \mathcal{G}_{\omega}\left(c_{2} \mathcal{G}_{\omega}^{*}(\mathbf{u})\right) \rightarrow-\nabla\left(c_{2} \nabla \cdot \mathbf{u}\right) \\
& \mathcal{C}_{\omega}\left(\mathbf{C}_{1} \cdot \mathcal{C}_{\omega}^{*}(\mathbf{u})\right) \rightarrow \nabla \times\left(\mathbf{C}_{1} \cdot(\nabla \times \mathbf{u})\right),
\end{aligned}
$$

where the convergence as $\varepsilon \rightarrow 0$ is with respect to $H^{-1}\left(\mathbb{R}^{n}\right)$.

Proof. Using Proposition 5.4 and a similar method of proof as for Lemma 5.6, the results are obtained.

We also have the following result.

Lemma 5.2. Let $\mathcal{L}$ denote a linear operator that commutes with the differential and nonlocal operators. Then, if $\mathcal{L} \mathbf{u} \in\left[H^{1}\left(\mathbb{R}^{n}\right)\right]^{n}, \mathcal{L} u \in H^{1}\left(\mathbb{R}^{n}\right)$, and $\mathcal{L} \mathbf{u} \in\left[H^{1}\left(\mathbb{R}^{3}\right)\right]^{3}$ as need be, we have

$$
\begin{array}{ll}
\mathcal{D}_{\omega}(\mathcal{L} \mathbf{u}) \rightarrow \nabla \cdot \mathcal{L} \mathbf{u} & \mathcal{D}_{\omega}^{*}(\mathcal{L} u) \rightarrow-\nabla \mathcal{L} u \\
\mathcal{G}_{\omega}(\mathcal{L} u) \rightarrow \nabla \mathcal{L} u & \mathcal{G}_{\omega}^{*}(\mathcal{L} \mathbf{u}) \rightarrow-\nabla \cdot \mathcal{L} \mathbf{u} \\
\mathcal{C}_{\omega}(\mathcal{L} \mathbf{u}) \rightarrow \nabla \times \mathcal{L} \mathbf{u} & \mathcal{C}_{\omega}^{*}(\mathcal{L} \mathbf{u}) \rightarrow \nabla \times \mathcal{L} \mathbf{u},
\end{array}
$$

where the convergence as $\varepsilon \rightarrow 0$ is with respect to $L^{2}\left(\mathbb{R}^{n}\right)$. 
If $\mathcal{L}$ is selected as a differential operator with constant coefficients, or its formal inverse, we can observe convergence in either stronger or weaker norms of the weighted operators.

\subsection{A connection between the nonlocal and local Gauss theorems}

Two interesting lemmas found in Ref $14^{\mathrm{q}}$ allow us to make another connection between nonlocal and local operators or, more precisely, between the nonlocal Gauss theorem (4.3a) and the classical Gauss theorem.

Given a two-point function $\boldsymbol{\nu}: \mathbb{R}^{n} \times \mathbb{R}^{n} \rightarrow \mathbb{R}^{k}$, we define the vector-valued point function $\mathbf{q}(\mathbf{x}): \mathbb{R}^{n} \rightarrow \mathbb{R}^{k}$ by

$$
\mathbf{q}(\mathbf{x}):=-\int_{\mathbb{R}^{n}}(\mathbf{y}-\mathbf{x}) \psi(\mathbf{x}, \mathbf{y}-\mathbf{x}) d \mathbf{y},
$$

where, with $p(\mathbf{x}, \mathbf{y})=\left(\boldsymbol{\nu}+\boldsymbol{\nu}^{\prime}\right) \cdot \boldsymbol{\alpha}$ and $\mathbf{z}=\mathbf{y}-\mathbf{x}$, the function $\psi: \mathbb{R}^{n} \times \mathbb{R}^{n} \rightarrow \mathbb{R}$ is given by

$$
\psi(\mathbf{x}, \mathbf{z})=\int_{0}^{1} p(\mathbf{x}+\lambda \mathbf{z}, \mathbf{x}-(1-\lambda) \mathbf{z}) d \lambda .
$$

Then, Lemmas I and II in Ref. 14 state that

$$
\nabla \cdot \mathbf{q}(\mathbf{x})=\int_{\mathbb{R}^{n}}\left(\boldsymbol{\nu}+\boldsymbol{\nu}^{\prime}\right) \cdot \boldsymbol{\alpha} d \mathbf{y} \quad \forall \mathbf{x} \in \mathbb{R}^{n}
$$

and

$$
\int_{\partial \Omega_{s}} \mathbf{q}(\mathbf{x}) \cdot \mathbf{n} d A=\int_{\Omega_{s}} \int_{\mathbb{R}^{n} \backslash \Omega_{s}}\left(\boldsymbol{\nu}+\boldsymbol{\nu}^{\prime}\right) \cdot \boldsymbol{\alpha} d \mathbf{y} d \mathbf{x}
$$

respectively, where $\mathbf{n}$ denotes the outward pointing unit normal vector along $\partial \Omega_{s}$. From (3.1a) and (5.18) we then have that

$$
\nabla \cdot \mathbf{q}=\mathcal{D}(\boldsymbol{\nu})
$$

and from (4.2a) and (5.19), using the by now familiar sequence of steps, we have that

$$
\begin{aligned}
\int_{\partial \Omega_{s}} \mathbf{q} \cdot \mathbf{n} d A & =\int_{\Omega_{s}} \int_{\mathbb{R}^{n} \backslash \Omega_{s}}\left(\boldsymbol{\nu}+\boldsymbol{\nu}^{\prime}\right) \cdot \boldsymbol{\alpha} d \mathbf{y} d \mathbf{x} \\
& =\int_{\Omega_{s}} \int_{\Omega_{c}}\left(\boldsymbol{\nu}+\boldsymbol{\nu}^{\prime}\right) \cdot \boldsymbol{\alpha} d \mathbf{y} d \mathbf{x}=\int_{\Omega_{s}} \mathcal{N}(\boldsymbol{\nu}) d \mathbf{x} .
\end{aligned}
$$

Then, the nonlocal Gauss theorem (4.3a) for $\boldsymbol{\nu}$, (5.20), and (5.21) imply that

$$
0=\int_{\Omega_{s}} \mathcal{D}(\boldsymbol{\nu}) d \mathbf{x}-\int_{\Omega_{s}} \mathcal{N}(\boldsymbol{\nu}) d \mathbf{x}=\int_{\Omega_{s}} \nabla \cdot \mathbf{q} d \mathbf{x}-\int_{\partial \Omega_{s}} \mathbf{q} \cdot \mathbf{n} d A,
$$

i.e., the classical Gauss's theorem for the vector-valued function q. Thus, we have shown that the nonlocal Gauss's theorem (4.3a) for the nonlocal vector two-point

${ }^{\mathrm{q}}$ See Ref. 12 for an English translation of Ref. 14. 
function $\boldsymbol{\nu}(\mathbf{x}, \mathbf{y})$ formally implies the classical Gauss theorem for the local vector point function $\mathbf{q}(\mathbf{x})$ derived from $\boldsymbol{\nu}$ through (5.17). Evidently, the Gauss theorem can be given a meaning without the notions of the divergence operator, unit normal vector, or surface.

\section{Examples of nonlocal volume-constrained problems}

The nonlocal point operators, the corresponding nonlocal adjoint operators, and the corresponding nonlocal interaction operators given in (3.1), (3.4), and (4.2), respectively, can be used to define nonlocal "boundary-value" problems that are analogous to classical boundary-value problems for partial differential equations. Here, we merely state problems involving scalar and vector "second-order" operators so that we are in the setting of "elliptic" problems. Specifically, we define nonlocal problems that are are analogous to the second-order differential boundary-value problems

$$
\begin{aligned}
& \left\{\begin{aligned}
-\nabla \cdot\left(\mathbf{C}_{2} \cdot \nabla u\right) & =f & & \text { in } \Omega \\
u & =g & & \text { on } \partial \Omega_{d} \\
\left(\mathbf{C}_{2} \cdot \nabla u\right) \cdot \mathbf{n} & =h & & \text { on } \partial \Omega_{n}
\end{aligned}\right. \\
& \left\{\begin{aligned}
-\nabla \cdot\left(\mathbf{C}_{4}: \nabla \mathbf{u}\right) & =\mathbf{f} & & \text { in } \Omega \\
\mathbf{u} & =\mathbf{g} & & \text { on } \partial \Omega_{d} \\
\left(\mathbf{C}_{4}: \nabla \mathbf{u}\right) \cdot \mathbf{n} & =\mathbf{h} & & \text { on } \partial \Omega_{n}
\end{aligned}\right.
\end{aligned}
$$

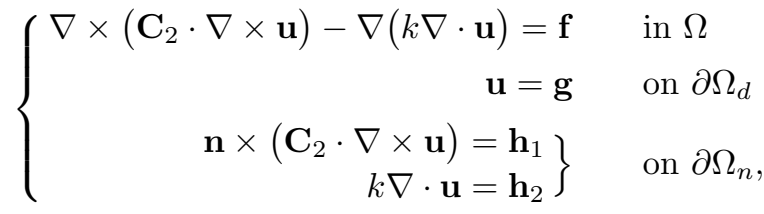

respectively, where $\partial \Omega=\partial \Omega_{d} \cup \partial \Omega_{n}$ denotes the boundary of $\Omega$ with $\partial \Omega_{d} \cap \partial \Omega_{n}=\emptyset$; $\partial \Omega_{d}$ and $\partial \Omega_{n}$ are the parts of the boundary $\partial \Omega$ on which Dirichlet and Neumann boundary conditions are applied, respectively. In (6.1a)-(6.1c), $\mathbf{C}_{4}, \mathbf{C}_{2}$, and $c$ denote fourth-order tensor, second-order tensor, and scalar point functions, respectively.

Each of the problems (6.1a)-(6.1c) for an unknown function, e.g., $u$ in (6.1a), consists of a partial differential equation and boundary conditions, where the latter may be viewed as constraints placed on possible solutions of the partial differential equation. Thus, in the case of local operators, constraints are applied along the boundary $\partial \Omega$ of the domain $\Omega$ on which the partial differential equation is applied. A consequence of the nonlocality of operators is that constraints analogous to the boundary conditions in (6.1a)-(6.1c) are applied instead over sets with positive measure in $\mathbb{R}^{n}$. Thus, in the case of nonlocal operators, constraints are applied on the interaction domain $\Omega_{c}$ that has positive volume and that corresponds to the domain $\Omega_{s}$ on which the nonlocal operator equation is applied. As a result, we refer 
to nonlocal problems with constraints imposed on volumes having positive volume as volume-constrained problems in contrast to local problems with constraints imposed on boundary surfaces that are universally referred to as boundary-value problems.

We now describe the nonlocal problems analogous to (6.1a)-(6.1c). In the differential equation setting we had to divide the boundary $\partial \Omega$ in the two parts $\partial \Omega_{d}$ and $\partial \Omega_{n}$ over which we applied Dirichlet and Neumann conditions, respectively. Similarly, in the nonlocal case we divide the interaction domain $\Omega_{c}$ into two subdomains that we use to apply Dirichlet-like and Neumann-like volume constraints. Let $\Omega_{c}=\Omega_{c d} \cup \Omega_{c n}$, where $\Omega_{c d} \cap \Omega_{c n}=\emptyset$ although either $\Omega_{c d}$ or $\Omega_{c n}$ may be empty. Over $\Omega_{c d}$ we specify function values, e.g., for a given function $g$, we set

$$
u(\mathbf{x})=g(\mathbf{x}) \quad \text { for } \mathbf{x} \in \Omega_{c d}
$$

which is, of course, a straightforward generalization of the Dirichlet boundary condition $u(\mathbf{x})=g(\mathbf{x})$ for $\mathbf{x} \in \partial \Omega_{d}$ for the partial differential equation case; see, e.g., (6.1a). The Neumann boundary condition in (6.1a) specifies $\mathbf{q} \cdot \mathbf{n}=\left(\mathbf{C}_{2} \cdot \nabla u\right) \cdot \mathbf{n}$ for $\mathbf{x} \in \partial \Omega_{n}$, i.e., the flux density our of $\Omega$ through $\mathbf{x} \in \partial \Omega_{n}$ is specified. For the nonlocal case we similarly specify the nonlocal flux density out of $\Omega_{s}$ into $\Omega_{c n}$, i.e., for a given function $h(\mathbf{x})$ and tensor function $\Theta_{2}(\mathbf{x}, \mathbf{y})$, we set

$$
\mathcal{N}_{c n}(\boldsymbol{\nu})(\mathbf{x})=\int_{\Omega_{c n}}\left(\boldsymbol{\nu}+\boldsymbol{\nu}^{\prime}\right) \cdot \boldsymbol{\alpha} d A=h(\mathbf{x}) \quad \text { for } \mathbf{x} \in \Omega_{s} \quad \text { with } \boldsymbol{\nu}=\mathbf{\Theta}_{2} \cdot \mathcal{D}^{*}(u)
$$

To define volume-constrained problems analogous to the boundary-value problems (6.1a)-(6.1c), we let $\boldsymbol{\Theta}_{4}, \boldsymbol{\Theta}_{2}$, and $\theta$ denote fourth-order tensor, second-order tensor, and scalar two-point functions, respectively, where the tensors are symmteric in the function and matrix senses and are positive definite. Using (6.2) and (6.3), the nonlocal volume-constrained problems corresponding to the boundaryvalue problems (6.1a)-(6.1c) are then given by

$$
\begin{gathered}
\left\{\begin{aligned}
\mathcal{D}\left(\boldsymbol{\Theta}_{2} \cdot \mathcal{D}^{*}(u)\right)=f & \text { in } \Omega_{s} \\
u=g & \text { in } \Omega_{c d} \\
\mathcal{N}_{\Omega_{c n}}\left(\boldsymbol{\Theta}_{2} \cdot \mathcal{D}^{*}(u)\right)=h & \text { in } \Omega_{s}
\end{aligned}\right. \\
\left\{\begin{aligned}
\mathcal{D}_{t}\left(\boldsymbol{\Theta}_{4}: \mathcal{D}_{t}^{*}(\mathbf{u})\right)=\mathbf{f} & \text { in } \Omega \\
\mathbf{u}=\mathbf{g} & \text { in } \Omega_{c d} \\
\mathcal{N}_{\Omega_{c n}, t}\left(\boldsymbol{\Theta}_{4}: \mathcal{D}_{t}^{*}(\mathbf{u})\right)=\mathbf{h} & \text { in } \Omega_{s}
\end{aligned}\right. \\
\left\{\begin{aligned}
\mathcal{C}\left(\boldsymbol{\Theta}_{2} \cdot \mathcal{C}^{*}(\mathbf{u})\right)+\mathcal{G}\left(\theta \mathcal{G}^{*}(\mathbf{u})\right)=\mathbf{f} & \text { in } \Omega_{s} \\
\mathbf{u}=\mathbf{g} & \text { in } \Omega_{c d} \\
\mathcal{T}_{\Omega_{c n}}\left(\boldsymbol{\Theta}_{2} \cdot \mathcal{C}^{*}(\mathbf{u})\right)+\mathcal{S}_{\Omega_{c n}}\left(\theta \mathcal{G}^{*}(\mathbf{u})\right)=\mathbf{h} & \text { in } \Omega_{s},
\end{aligned}\right.
\end{gathered}
$$

respectively.

The nonlocal Green's first identities (4.2) are useful for defining variation formulations of the nonlocal volume-constrained problems. For example, from (4.8a) 
and (6.4a), we have that $u(\mathbf{x})$ satisfies $u=g$ on $\Omega_{c d}$, and for suitable test functions $v(\mathbf{x})$ that vanish on $\Omega_{c d}$,

$$
\int_{\Omega_{s}} \int_{\Omega_{s}} \mathcal{D}^{*}(u) \cdot \mathbf{\Theta}_{2} \cdot \mathcal{D}^{*}(v) d \mathbf{y} d \mathbf{x}=\int_{\Omega_{s}} v f d \mathbf{x}-\int_{\Omega_{s}} v h d \mathbf{x} .
$$

The kernel function $\boldsymbol{\alpha}$ dictates the choice of function spaces for the trial and test functions $u$ and $v$, respectively, so that the problem (6.5) is well posed.

A special form of the nonlocal volume-constrained problem (6.4a) corresponding to a scalar-valued solution is studied in Ref. 9 by appealing to a variational formulation. Well-posedness results are provided in Ref. 9 for the case in which the natural energy space (used to define the variational problem) is equivalent to $L^{2}(\Omega)$, the space of square integrable functions. Although the notion of a nonlocal vector calculus is not introduced, the recent book Ref. 2 describes, using semigroup analysis, substantial recent work on nonlocal diffusion and its relationship to (6.1a). The report Ref. 5 extends these previous results to the case when the natural energy space is equivalent a fractional Sobolev space, a proper subspace of $L^{2}(\Omega)$. In this case, the variational problem possess smoothing properties akin to those for elliptic partial differential equations but with possibly reduced order.

\section{Local and nonlocal balance laws}

Along with kinematics and constitutive relations, balance laws are a cornerstone of continuum mechanics. A balance law postulates that the rate of change of the amount of a quantity in any subregion of a body is given by the rate at which that quantity is produced within the subregion minus the rate at which the quantity exits the subregion; the latter is referred to as the flux out of the subregion. In the classical differential equation setting of continuum mechanics, the flux is local, i.e., the quantity exits or enters the subregion through its boundary. Difficulties arise, however, in the classical setting due to, e.g., shock waves, corner singularities, and material failure, all of which are troublesome when defining an appropriate notion for the "flux through the boundary of a subregion."

The nonlocal vector calculus we develop has an important application to nonlocal balance laws for which subregions not in direct contact may have a non-zero interaction, i.e., there is a nonzero flux between the subregions. This is accomplished by defining the flux in terms of interactions between disjoint open regions of positive measure that are possibly a finite distance apart. An important feature of nonlocal balance laws is that the significant technical details associated with determining normal and tangential traces along boundaries of suitable regions is obviated when fluxes are induced through interactions between volumes. Our nonlocal calculus, then, provides an alternative to standard approaches for circumventing the tech-

nicalities associated with lack of sufficient regularity in local balance laws such as measure-theoretic generalizations of the Gauss-Green theorem (see, e.g., Refs. 3 and 16) or the use of the fractional calculus (see, e.g., Refs. 1 and 21). 


\subsection{Abstract balance laws}

We start with an abstract balance law for an open subset $\Omega_{s} \subseteq \mathbb{R}^{n}$ given by

$$
\mathcal{A}(\Omega ; q)=\mathcal{P}(\Omega)-\mathcal{I}\left(\Omega, \mathbb{R}^{n} \backslash \Omega ; q\right) \quad \forall \Omega \subset \Omega_{s}
$$

which postulates that $\mathcal{A}(\Omega ; q)$ (the rate of change of the amount of the intensive quantity $q$ in any open subregion $\Omega \subset \Omega_{s}$ ) is equal to $\mathcal{P}(\Omega)$ (the rate at which the intensive quantity is produced in the subregion by external sources) minus $\mathcal{I}\left(\Omega, \mathbb{R}^{n} \backslash \Omega ; q\right)$ (the rate at which the intensive quantity exits the subregion). In (7.1), $q(\mathbf{x}, t)$ may be a scalar or vector time-dependent point function and we assume that $\mathcal{P}(\Omega)$, the rate of production of $q$ in any subregion $\Omega$ is given, e.g., through the specification of an external source function. Here, we take the usual choices

$$
\mathcal{A}(\Omega ; q)=\frac{\partial}{\partial t} \int_{\Omega} q(\mathbf{x}, t) d \mathbf{x} \quad \text { and } \quad \mathcal{P}(\Omega)=\int_{\Omega} b(\mathbf{x}, t) d \mathbf{x}
$$

for the rate of change and production terms, respectively, where $b(\mathbf{x}, t)$ denotes a given function. Note that if we assume the general case for which points in $\Omega_{s}$ only interact with points in $\Omega_{c} \subseteq \mathbb{R}^{n} \backslash \Omega_{s}$, then (7.1) reduces to

$$
\mathcal{A}(\Omega ; q)=\mathcal{P}(\Omega)-\mathcal{I}\left(\Omega, \Omega_{c} ; q\right) \quad \forall \Omega \subset \Omega_{s}
$$

We assume that the interaction or flux operator $\mathcal{I}(\cdot, \cdot ; q)$ in $(7.1)$ or $(7.3)$ is alternating, i.e.,

$$
\mathcal{I}(\Omega, \Omega ; q)=0 \quad \forall \Omega \subseteq \mathbb{R}^{n}
$$

and that

$$
\left.\begin{array}{l}
\mathcal{I}\left(\Omega_{1} \cup \Omega_{2}, \Omega_{3} ; q\right) \\
\quad=\mathcal{I}\left(\Omega_{1}, \Omega_{3} ; q\right)+\mathcal{I}\left(\Omega_{2}, \Omega_{3} ; q\right)-\mathcal{I}\left(\Omega_{1} \cap \Omega_{2}, \Omega_{3} ; q\right) \\
\quad \mathcal{I}\left(\Omega_{3}, \Omega_{1} \cup \Omega_{2} ; q\right) \\
\quad=\mathcal{I}\left(\Omega_{3}, \Omega_{1} ; q\right)+\mathcal{I}\left(\Omega_{3}, \Omega_{2} ; q\right)-\mathcal{I}\left(\Omega_{3}, \Omega_{1} \cap \Omega_{2} ; q\right)
\end{array}\right\} \quad \forall \Omega_{1}, \Omega_{2}, \Omega_{3} \subseteq \mathbb{R}^{n}
$$

The assumed properties $(7.4)$ and $(7.5)$ of $\mathcal{I}(\cdot, \cdot ; q)$ immediately imply a third property.

Proposition 7.1. Assume the interaction operator $\mathcal{I}(\cdot, \cdot)$ satisfies (7.4) and (7.8). Then, that operator is antisymmetric, i.e., we have

$$
\mathcal{I}\left(\Omega_{1}, \Omega_{2} ; q\right)+\mathcal{I}\left(\Omega_{2}, \Omega_{1} ; q\right)=0 \quad \forall \Omega_{1}, \Omega_{2} \subseteq \mathbb{R}^{n} .
$$


Proof. Through repeated applications of (7.4) and (7.5), we obtain

$$
\begin{aligned}
0= & \mathcal{I}\left(\Omega_{1} \cup \Omega_{2}, \Omega_{1} \cup \Omega_{2} ; q\right) \\
= & \mathcal{I}\left(\Omega_{1}, \Omega_{1} \cup \Omega_{2} ; q\right)+\mathcal{I}\left(\Omega_{2}, \Omega_{1} \cup \Omega_{2} ; q\right)-\mathcal{I}\left(\Omega_{1} \cap \Omega_{2}, \Omega_{1} \cup \Omega_{2} ; q\right) \\
= & \mathcal{I}\left(\Omega_{1}, \Omega_{1} ; q\right)+\mathcal{I}\left(\Omega_{1}, \Omega_{2} ; q\right)-\mathcal{I}\left(\Omega_{1}, \Omega_{1} \cap \Omega_{2} ; q\right) \\
& \quad+\mathcal{I}\left(\Omega_{2}, \Omega_{1} ; q\right)+\mathcal{I}\left(\Omega_{2}, \Omega_{2} ; q\right)-\mathcal{I}\left(\Omega_{2}, \Omega_{1} \cap \Omega_{2} ; q\right) \\
& \quad-\quad \mathcal{I}\left(\Omega_{1} \cap \Omega_{2}, \Omega_{1} ; q\right)-\mathcal{I}\left(\Omega_{1} \cap \Omega_{2}, \Omega_{2} ; q\right)+\mathcal{I}\left(\Omega_{1} \cap \Omega_{2}, \Omega_{1} \cap \Omega_{2} ; q\right) \\
= & \mathcal{I}\left(\Omega_{1}, \Omega_{2} ; q\right)+\mathcal{I}\left(\Omega_{2}, \Omega_{1} ; q\right)-\mathcal{I}\left(\Omega_{1}, \Omega_{1} \cap \Omega_{2} ; q\right)-\mathcal{I}\left(\Omega_{1} \cap \Omega_{2}, \Omega_{1} ; q\right) \\
& \quad-\mathcal{I}\left(\Omega_{2}, \Omega_{1} \cap \Omega_{2} ; q\right)-\mathcal{I}\left(\Omega_{1} \cap \Omega_{2}, \Omega_{2} ; q\right) \\
= & \mathcal{I}\left(\Omega_{1}, \Omega_{2} ; q\right)+\mathcal{I}\left(\Omega_{2}, \Omega_{1} ; q\right) \\
& \quad-\mathcal{I}\left(\Omega_{1} \backslash\left(\Omega_{1} \cap \Omega_{2}\right), \Omega_{1} \cap \Omega_{2} ; q\right)-\mathcal{I}\left(\Omega_{1} \cap \Omega_{2}, \Omega_{1} \cap \Omega_{2} ; q\right) \\
& \quad-\quad \mathcal{I}\left(\Omega_{1} \cap \Omega_{2}, \Omega_{1} \backslash\left(\Omega_{1} \cap \Omega_{2}\right) ; q\right)-\mathcal{I}\left(\Omega_{1} \cap \Omega_{2}, \Omega_{1} \cap \Omega_{2} ; q\right) \\
& \quad-\mathcal{I}\left(\Omega_{2} \backslash\left(\Omega_{1} \cap \Omega_{2}\right), \Omega_{1} \cap \Omega_{2} ; q\right)-\mathcal{I}\left(\Omega_{1} \cap \Omega_{2}, \Omega_{1} \cap \Omega_{2} ; q\right) \\
& \quad-\mathcal{I}\left(\Omega_{1} \cap \Omega_{2}, \Omega_{2} \backslash\left(\Omega_{1} \cap \Omega_{2}\right) ; q\right)-\mathcal{I}\left(\Omega_{1} \cap \Omega_{2}, \Omega_{1} \cap \Omega_{2} ; q\right) \\
= & \mathcal{I}\left(\Omega_{1}, \Omega_{2} ; q\right)+\mathcal{I}\left(\Omega_{2}, \Omega_{1} ; q\right) \\
& \quad-\mathcal{I}\left(\Omega_{1} \backslash\left(\Omega_{1} \cap \Omega_{2}\right), \Omega_{1} \cap \Omega_{2} ; q\right)-\mathcal{I}\left(\Omega_{1} \cap \Omega_{2}, \Omega_{1} \backslash\left(\Omega_{1} \cap \Omega_{2}\right) ; q\right) \\
& \quad-\mathcal{I}\left(\Omega_{2} \backslash\left(\Omega_{1} \cap \Omega_{2}\right), \Omega_{1} \cap \Omega_{2} ; q\right)-\mathcal{I}\left(\Omega_{1} \cap \Omega_{2}, \Omega_{2} \backslash\left(\Omega_{1} \cap \Omega_{2}\right) ; q\right) .
\end{aligned}
$$

Now, assume that the regions $\Omega_{1}$ and $\Omega_{2}$ are disjoint, i.e., $\Omega_{1} \cap \Omega_{2}=\emptyset$. Then, (7.6) immediately follows from (7.7). Thus, we have proven (7.6) for disjoint regions.

If $\Omega_{1} \cap \Omega_{2} \neq \emptyset$, we have that $\Omega_{1} \backslash\left(\Omega_{1} \cap \Omega_{2}\right)$ and $\Omega_{1} \cap \Omega_{2}$ are disjoint regions as are $\Omega_{2} \backslash\left(\Omega_{1} \cap \Omega_{2}\right)$ and $\Omega_{1} \cap \Omega_{2}$. Then, from (7.6) for disjoint regions, which we just proved, and (7.7), we obtain (7.6) for the case $\Omega_{1} \cap \Omega_{2} \neq \emptyset$.

The following result, which holds only for disjoint subsets, immediately follows from (7.5).

Proposition 7.2. Assume the interaction operator $\mathcal{I}(\cdot, \cdot)$ satisfies (7.4) and (7.5). Then, $\mathcal{I}(\cdot, \cdot ; q)$ is bilinear for disjoint subsets, i.e.,

$$
\left.\begin{array}{l}
\mathcal{I}\left(\Omega_{1} \cup \Omega_{2}, \Omega_{3} ; q\right)=\mathcal{I}\left(\Omega_{1}, \Omega_{3} ; q\right)+\mathcal{I}\left(\Omega_{2}, \Omega_{3} ; q\right) \\
\mathcal{I}\left(\Omega_{3}, \Omega_{1} \cup \Omega_{2} ; q\right)=\mathcal{I}\left(\Omega_{3}, \Omega_{1} ; q\right)+\mathcal{I}\left(\Omega_{3}, \Omega_{2} ; q\right)
\end{array}\right\} \quad \forall \Omega_{1}, \Omega_{2}, \Omega_{3} \subseteq \mathbb{R}^{n} \text { such }
$$

The assumption (7.4) postulates that there are no self-interactions. The result (7.6) is an abstract action-reaction principle, i.e., the flux from $\Omega_{1}$ into $\Omega_{2}$ is equal and opposite to the flux from $\Omega_{2}$ into $\Omega_{1}$. The result (7.8) states that the total interaction or flux from two disjoint regions $\Omega_{1}$ and $\Omega_{2}$ into a region $\Omega_{3}$ is simply the sum of the individual fluxes from $\Omega_{1}$ into $\Omega_{3}$ and from $\Omega_{2}$ into $\Omega_{3} ;(7.8)$ also states that the total flux from a region $\Omega_{3}$ into two disjoint regions $\Omega_{1}$ and $\Omega_{2}$ is the sum of the individual fluxes from $\Omega_{3}$ into the two subregions. 


\subsubsection{Abstract local balance laws}

For disjoint open regions $\Omega_{1}, \Omega_{2} \subset \mathbb{R}^{n}$, we assume that the interaction operator $\mathcal{I}\left(\Omega_{1}, \Omega_{2} ; q\right)$ is given by $(2.1)$ so that

$$
\mathcal{I}_{l o c}\left(\Omega_{1}, \Omega_{2} ; q\right)=\int_{\partial \Omega_{12}} \mathbf{q} \cdot \mathbf{n} d A \quad \text { for } \Omega_{1} \cap \Omega_{2}=\emptyset \quad \text { with } \quad \partial \Omega_{12}=\bar{\Omega}_{1} \cap \bar{\Omega}_{2}
$$

for some vector $\mathbf{q}$, where $\partial \Omega_{12}$ denotes the common boundary of $\Omega_{1}$ and $\Omega_{2}$ and $\mathbf{n}$ the unit normal along $\partial \Omega_{12}$ pointing out of $\Omega_{1}$. Note that

$$
\text { if } \partial \Omega_{12}=\emptyset, \text { then } \mathcal{I}_{l o c}\left(\Omega_{1}, \Omega_{2} ; q\right)=0 .
$$

The interaction operator given in (7.9) satisfies (7.8) and, by (2.2), is antisymmetric.

From $(7.1),(7.2),(7.9)$, and the Gauss theorem, we have that

$$
\int_{\Omega}\left(q_{t}+\nabla \cdot \mathbf{q}-b\right) d \mathbf{x}=0 \quad \forall \Omega \subset \Omega_{s}
$$

from which, because $\Omega$ is arbitrary in $\Omega_{s}$, we obtain the local field equation

$$
q_{t}+\nabla \cdot \mathbf{q}=b \quad \forall \mathbf{x} \in \Omega_{s}
$$

corresponding to the balance law (7.1) and the interaction operator (7.9).

To obtain a field equation in terms of the intensive variable $q$, a constitutive equation must be postulated relating the flux vector $\mathbf{q}$ to the intensive variable $q$. For example, consider the case of heat conduction for which the variable $q$ denotes the temperature. Then, a constitutive equation relating the heat flux vector $\mathbf{q}$ to the temperature $q$ is given by the Fourier heat law $\mathbf{q}=-\kappa \nabla q$, where $\kappa$ denotes the thermal diffusivity. Then, from (7.10), we have the field equation

$$
q_{t}=\kappa \Delta q+b
$$

for the temperature $q$, i.e., the heat equation.

\subsubsection{Abstract nonlocal balance laws}

From (2.3), in the nonlocal case, we define the interaction operator ${ }^{\mathrm{r}}$

$$
\begin{aligned}
\mathcal{I}_{\text {nonloc }}\left(\Omega_{1}, \Omega_{2} ; q\right) & :=\int_{\Omega_{1}} \mathcal{N}_{\Omega_{2}}(\boldsymbol{\nu}) d \mathbf{x} \\
& =\int_{\Omega_{1}} \int_{\Omega_{2}}\left(\boldsymbol{\nu}+\boldsymbol{\nu}^{\prime}\right) \cdot \boldsymbol{\alpha} d \mathbf{y} d \mathbf{x} \quad \forall \Omega_{1}, \Omega_{2} \subset \mathbb{R}^{n}
\end{aligned}
$$

that gives the nonlocal flux from $\Omega_{1}$ into $\Omega_{2}$; in (7.12), $\mathcal{N}_{\Omega_{2}}(\boldsymbol{\nu}): \Omega_{1} \rightarrow \mathbb{R}$ denotes an interaction density. ${ }^{\text {s }}$ The interaction operator given in (7.12) satisies (7.8), i.e.,

${ }^{\mathrm{r}}$ Comparing $\mathcal{I}_{\text {loc }}\left(\Omega_{1}, \Omega_{2} ; q\right)$ and $\mathcal{I}_{\text {nonloc }}\left(\Omega_{1}, \Omega_{2} ; q\right)$, we see that the role of the flux density $\mathbf{q} \cdot \mathbf{n}$ for the local balance is now assumed by the nonlocal flux density $\mathcal{N}(\boldsymbol{\nu})$. Just as was the case for q, $\boldsymbol{\nu}$ should be related to the intensive variable $q$ through a constitutive law.

${ }^{\mathrm{s}}$ See also page 85 in Ref. 20 and page 29 in 15 . 
is bilinear for disjoint regions, and, because $\boldsymbol{\alpha}(\mathbf{x}, \mathbf{y})$ is antisymmetric, is alternating and therefore antisymmetric.

In stark contrast to that for the interaction operator $\mathcal{I}_{\text {loc }}(\cdot, \cdot ; q)$ given in $(7.9)$, the flux induced by $\mathcal{I}_{\text {nonloc }}(\cdot, \cdot ; q)$ dispenses with the need for determining a unit normal to an orientable surface and instead considers volume interactions among regions. Such a relation and (7.12) can also be interpreted more broadly. Furthermore, the local interaction operator $\mathcal{I}_{l o c}(\cdot, \cdot ; q)$ vanishes whenever $\bar{\Omega}_{1} \cap \bar{\Omega}_{2}=\emptyset$ whereas, in general, the nonlocal interaction operator $\mathcal{I}_{\text {nonloc }}(\cdot, \cdot ; q)$ does not.

We derive a nonlocal field equation analogous to the classical heat equation. Due to (7.4), we have that

$$
\mathcal{I}_{\text {nonloc }}\left(\Omega, \Omega_{c}\right)=\int_{\Omega} \mathcal{N}(\boldsymbol{\nu}) d \mathbf{x}=\int_{\Omega} \int_{\Omega_{c}}\left(\boldsymbol{\nu}+\boldsymbol{\nu}^{\prime}\right) \cdot \boldsymbol{\alpha} d \mathbf{y} d \mathbf{x} \quad \forall \Omega \subset \Omega_{s}
$$

that we substitute, along with (7.2), into (7.1) to obtain

$$
\int_{\Omega}\left(q_{t}+\mathcal{N}(\boldsymbol{\nu})-b\right) d \mathbf{x} \quad \forall \Omega \subset \Omega_{s} .
$$

Then, using the nonlocal Gauss theorem (4.3a), we obtain

$$
\int_{\Omega}\left(q_{t}+\mathcal{D}(\boldsymbol{\nu})-b\right) d \mathbf{x} \quad \forall \Omega \subset \Omega_{s}
$$

from which it follows that, because $\Omega$ is arbitrary in $\Omega_{s}$,

$$
q_{t}+\mathcal{D}(\boldsymbol{\nu})=b \quad \forall \mathbf{x} \in \Omega_{s} .
$$

The interaction vector $\boldsymbol{\nu}$ is related to the extensive quantity $q$ through the constitutive relation $\boldsymbol{\nu}=\kappa \mathcal{D}^{*}(q)$, where the adjoint operator $\mathcal{D}^{*}$ is defined in (3.4a). We then obtain the field equation for $q$ given by

$$
q_{t}+\kappa \mathcal{D D}^{*}(q)=b \quad \forall \mathbf{x} \in \Omega_{s}
$$

which may be viewed as a nonlocal heat equation analogous to the classical local heat equation (7.11). Note that that the operator $\kappa \mathcal{D} \mathcal{D}^{*}(\cdot)$ is exactly that that appears in the steady-state volume constrained problem (6.4a) with $\boldsymbol{\Theta}_{2}=\kappa \mathbf{I}$, where $\kappa$ is constant and $\mathbf{I}$ denotes the identity tensor.

\subsection{The peridynamics nonlocal theory of continuum mechanics}

We now demonstrate that the nonlocal calculus developed in the earlier sections is synergistic with the peridynamic nonlocal theory of continuum mechanics.

The abstract balance law (7.1) and the discussion that ensues generalize, in straightforward manner, to vector valued intensive quantities. In particular, we obtain the field equation

$$
\frac{\partial \mathbf{m}}{\partial t}+\mathcal{D}_{t}(\mathbf{\Psi})=\mathbf{b} \quad \forall \mathbf{x} \in \Omega_{s},
$$

where the operator $\mathcal{D}_{t}$ is defined in (3.8a) and the tensor $\boldsymbol{\Psi}$ is related to $\mathbf{m}$ through a constitutive relation. In particular, consider the case of $\mathbf{m}$ denoting the momentum 
density for a continuum solid material so that $\mathbf{m}=\rho(\mathbf{x}) \frac{\partial}{\partial t} \mathbf{u}(\mathbf{x}, t)$, where $\rho(\cdot)$ denotes the material density and $\mathbf{u}(\mathbf{x}, t)$ the displacement. A constitutive equation is given by $\Psi(\mathbf{x}, \mathbf{y})=-\frac{1}{2}\left(\mathcal{D}_{t}^{*}(\mathbf{u})\right)^{T}$, where the adjoint operator $\mathcal{D}_{t}^{*}(\cdot)$ is defined in $(3.8 \mathrm{~b})$. We then have, from (7.13), that

$$
\rho \frac{\partial^{2} \mathbf{u}}{\partial t^{2}}+\frac{1}{2} \mathcal{D}_{t}\left(\left(\mathcal{D}_{t}^{*} \mathbf{u}\right)^{T}\right)=\mathbf{b} \quad \forall \mathbf{x} \in \Omega_{s}
$$

Substituting the definitions of $\mathcal{D}_{t}$ and $\mathcal{D}_{t}^{*}$, we obtain, invoking (4.1),

$$
\mathcal{D}_{t}\left(\left(\mathcal{D}_{t}^{*} \mathbf{u}\right)^{T}\right)=-2 \int_{\Omega_{s c}}(\boldsymbol{\alpha} \otimes \boldsymbol{\alpha}) \cdot\left(\mathbf{u}^{\prime}-\mathbf{u}\right) d \mathbf{x}
$$

so that, from (7.14), we have that

$$
\rho \frac{\partial^{2} \mathbf{u}}{\partial t^{2}}=\int_{\Omega_{s c}}(\boldsymbol{\alpha} \otimes \boldsymbol{\alpha}) \cdot\left(\mathbf{u}^{\prime}-\mathbf{u}\right) d \mathbf{x}+\mathbf{b} \quad \forall \mathbf{x} \in \Omega_{s} .
$$

A mechanical perspective indicates that $\left(\mathcal{D}_{t}^{*} \mathbf{u}\right)^{T}$ describes the deformation of $\mathbf{u}$ and that the constitutive relation maps the deformation to the force density given by the integral operator. Because the integrand of (7.15) is antisymmetric with respect to the arguments $\mathbf{x}$ and $\mathbf{y}$, the operator (7.15) induces an interaction, i.e., that of forces between subregions. ${ }^{\mathrm{t}}$

Equation (7.16) is a generalization of the conservation of momentum equation in the linearized bond-based peridynamic theory introduced in Ref. 17. In fact, if we require that the null space of the integral operator in (7.14) contains rigid motions, i.e., that

$$
\mathcal{D}_{t}\left(\left(\mathcal{D}_{t}^{*}(\mathbf{A x}+\mathbf{c})\right)^{T}\right)=\mathbf{0}
$$

for all constant skew-symmetric tensors $\mathbf{A}$ and all constant vectors $\mathbf{c}$,

then a sufficient condition is that $\boldsymbol{\alpha}(\mathbf{x}, \mathbf{y})=(\mathbf{y}-\mathbf{x}) \zeta(|\mathbf{y}-\mathbf{x}|)$, where $\zeta: \mathbb{R}^{+} \rightarrow \mathbb{R}$. Then, from (7.16), we have that

$$
\rho \frac{\partial^{2} \mathbf{u}}{\partial t^{2}}=\int_{\Omega_{s c}} \frac{(\mathbf{y}-\mathbf{x}) \otimes(\mathbf{y}-\mathbf{x})}{\sigma(|\mathbf{y}-\mathbf{x}|)} \cdot\left(\mathbf{u}^{\prime}-\mathbf{u}\right) d \mathbf{x}+\mathbf{b} \quad \forall \mathbf{x} \in \Omega_{s},
$$

where $\sigma:=\zeta^{-2}$, which is the linearized peridynamic bond-based model of Ref. 17 . In Ref. 6, analytical conditions on $\sigma$ describing the amount of smoothing associated with the integral operator are given and the well posedness of the balance of linear momentum is discussed. That paper also demonstrates that, for the case of localized kernels, as $\varepsilon \rightarrow 0$,

$$
-\frac{1}{2} \mathcal{D}_{t}\left(\left(\mathcal{D}_{t}^{*} \mathbf{u}\right)^{T}\right) \rightarrow-\mu \nabla \cdot(\nabla \mathbf{u})-2 \mu \nabla(\nabla \cdot \mathbf{u})
$$

${ }^{\mathrm{t}}$ In the local case, (7.9) is an abstraction of Cauchy's postulate in mechanics. There, the intensive variable $q$ is the vector momentum density and $\mathbf{q}$ is the stress tensor with $\mathbf{q} \cdot \mathbf{n}$ then being the stress force density at a point of a surface. Then, Cauchy's postulate states that the interaction between two abutting regions occurs at the common interface between the two regions and is given by the integral of the stress force along that interface. This is exactly a word description of (7.9). 
which is the Navier operator of linear elasticity for Poisson ratio one-quarter; see also Ref. 7. The subsequent paper Ref. 23 considers volume-constrained problems on bounded domains in $\mathbb{R}$ and squares in $\mathbb{R}^{2}$.

The state-based peridynamic theory ${ }^{19}$ requires the consideration of both volumetric and shear deformations. Similar to the above discussion for bond-based peridynamics models, we may also formulate the state-based peridynamic model in terms of the nonlocal operators. Let $\boldsymbol{\alpha}$ and $\omega$ be given by (5.9). Then, the linear state-based peridynamic integral operator ${ }^{18}$ is given by

$$
-\mathcal{D}_{t}\left(\eta\left(\mathcal{D}_{t}^{*} \mathbf{u}\right)^{T}\right)-\mathcal{D}_{t, \omega}\left(\lambda \operatorname{Tr}\left(\mathcal{D}_{t, \omega}^{*} \mathbf{u}\right) \mathbf{I}\right)
$$

where $\eta$ and $\lambda$ are materials constants and $\mathcal{D}_{t}, \mathcal{D}_{t}^{*}, \mathcal{D}_{t, w}$, and $\mathcal{D}_{t, w}^{*}$ are given by (3.8a), (3.8b), (5.6), and (5.7), respectively. The scalar $\operatorname{Tr}\left(\mathcal{D}_{t, \omega}^{*} \mathbf{u}\right)$ measures the volumetric change, or dilatation, in the material so that $\operatorname{Tr}\left(\mathcal{D}_{t, \omega}^{*} \mathbf{u}\right) \mathbf{I}$ is a diagonal tensor representing volumetric stress. This allows us to readily apply the nonlocal calculus to study the well-posedness of both free-space and volume-constrained linear peridynamic state-based balance laws, and also suggests why, in the limit as $\varepsilon \rightarrow 0$, the operator given by (7.18) leads to the linear Navier operator of elasticity for linear isotropic materials with general Poisson ratios, e.g., not relegated to a value of one-quarter. This is the subject of forthcoming research.

\section{Acknowledgments}

Q. Du was supported in part by the U.S. Department of Energy grant DESC0005346 and the U.S. National Science Foundation grant DMS-1016073.

M. Gunzburger was supported by the U.S. Department of Energy grant number DE-SC0004970 and the U.S. National Science Foundation grant DMS-1013845. Also supported in part while in residence at Oxford University under award number KUK-C1-013-04 made by King Abdullah University of Science and Technology (KAUST).

Sandia is a multiprogram laboratory operated by Sandia Corporation, a Lockheed Martin Company, for the U.S. Department of Energy under contract DE-AC0494AL85000. Supported in part by the U.S. Department of Energy grant number FWP-09-014290 through the Office of Advanced Scientific Computing Research, DOE Office of Science.

K. Zhou was supported in part by the U.S. Department of Energy grant DESC0005346 and the U.S. National Science Foundation grant DMS-1016073.

\section{References}

1. R. Almeida, A. Malinowska, and D. Torres, A fractional calculus of variations for multiple integrals with application to vibrating string, J. Math. Physics 51 (2010), 033503, doi:10.1063/1.3319559.

2. F. Andreu, J. Mazón, J. Rossi, and J. Toledo, Nonlocal diffusion problems, Mathematical Surveys and Monographs, 165, American Mathematical Society, 2010. 
3. G. Chen, M. Torres, and W. Ziemer, Gauss-Green theorem for weakly differentiable vector fields, sets of finite perimeter, and balance laws, Comm. Pure Appl. Math. 62 (2009), 242-304, doi:10.1002/cpa.20262.

4. T. Coulhon and A. Grigoryan, Random walks on graphs with regular volume growth, Geom. Funct. Anal. 8 (1998), 656-701, 10.1007/s000390050070.

5. Q. Du, M. Gunzburger, R. Lehoucq, and K. Zhou, Analysis and approximation of nonlocal diffusion problems with volume constraints, Technical Report 2011-3168J, Sandia National Laboratories, Albuquerque, (2011).

6. Q. Du and K. Zhou, Mathematical analysis for the peridynamic nonlocal continuum theory, Math. Model. Numer. Anal. 45 (2011), 217-234.

7. E. Emmrich and O. Weckner, On the well-posedness of the linear peridynamic model and its convergence towards the Navier equation of linear elasticity, Commun. Math. Sci. 5 (2007), 851-864.

8. G. Gilboa and S. Osher, Nonlocal operators with applications to image processing, Multi. Model. Simul. 7 (2008), 1005-1028.

9. M. Gunzburger and R. Lehoucq, A nonlocal vector calculus with application to nonlocal boundary value problems, Multi. Model. Simul. 8 (2010), 1581-1620, doi:10.1137/090766607.

10. L. Ignat and J. Rossi, Decay estimates for nonlocal problems via energy methods, $J$. Math. Pures Appl. 92 (2009), 163-187.

11. X. Jiang, L.-H. Lim, Y. Yao, and Y. Ye, Statistical ranking and combinatorial Hodge theory, Math. Prog. 127 (2011), 203-244, doi:10.1007/s10107-010-0419-x.

12. R. Lehoucq and O. von Lilienfeld, Translation of Walter Noll's "Derivation of the Fundamental Equations of Continuum Thermodynamics from Statistical Mechanics", J. Elast. 100 (2010), 5-24.

13. O. Lézoray, V.-T. Ta, and A. Elmoataz, Partial differences as tools for filtering data on graphs, Pattern Recog. Lett. 31 (2010), 2201-2213.

14. Walter Noll, Die Herleitung der Grundgleichungen der Thermomechanik der Kontinua aus der statistischen Mechanik, Indiana U. Math. J. 4 (1955), 627-646; original publishing journal was the J. Rat. Mech. Anal. See the English translation Ref. 12.

15. W. Noll, Thoughts on the concept of stress, J. Elast. 100 (2010), 25-32, doi:10.1007/s10659-010-9247-8.

16. F. Schuricht, A new mathematical foundation for contact interactions in continuum physics, Arch Rat. Mech. Anal. 184 (2007), 495-551, doi:10.1007/s00205-006-0032-6.

17. S. Silling, Reformulation of elasticity theory for discontinuities and long-range forces, J. Mech. Phys. Solids 48 (2000), 175-209.

18. L Linearized theory of peridynamic states, J. Elast. 99 (2010), 85-111.

19. S. Silling, M. Epton, O. Weckner, J. Xu, and E. Askari, Peridynamic states and constitutive modeling, J. Elast. 88 (2007), 151-184.

20. S. Silling and R. Lehoucq, Peridynamic theory of solid mechanics, Adv. Appl. Mech. 44 (2010), 73-69, doi:10.1016/S0065-2156(10)44002-8.

21. V. Tarasov, Fractional vector calculus and fractional Maxwell's equations, Annals Phys. 323 (2008), 2756-2778, doi:10.1016/j.aop.2008.04.005.

22. D. Zhou and B. Schölkopf, Regularization on discrete spaces, Pattern Recognition, Proceedings of the 27th DAGM Symposium (W.G Kropatsch, R. Sablatnig, and A. Hanbury, eds.), LNCS, 3663, Springer (2005), pp. 361-368.

23. K. Zhou and Q. Du, Mathematical and numerical analysis of linear peridynamic models with nonlocal boundary conditions, SIAM J. Numer. Anal. 48 (2010), 1759-1780, doi:10.1137/090781267. 



\section{RECENT REPORTS}

28/11 Growth of confined cancer spheroids: a combined experimental and mathematical modelling approach

Loessner

Flegg

Byrne

Hall

Moroney

Clements

McElwain

Hutmacher

29/11 Floating carpets and the delamination of elastic sheets

30/11 Numerical Study of Liquid Crystal Elastomers by a Mixed Finite

31/11 The indentation of pressurized elastic shells: From polymeric capsules to yeast cells

32/11 Wrinkling of pressurized elastic shells

33/11 Data assimilation using bayesian filters and B-spline geological models

Wagner

Vella

Luo

Calderer

Vella

Ajdari

Vaziri

Boudaoud

Vella

Ajdari

Vaziri

Boudaoud

Duan

Farmer

Hoteit

$\mathrm{Lu}$

Moroz

34/11 Review of nonlinear Kalman, ensemble and particle filtering with application to the reservoir history matching problem

Luo

Hoteit

Duan

Wang

35/11 Modelling a Tethered Mammalian Sperm Cell undergoing Hyperactivation

Curtis

Kirkman-Brown

Connolly

Gaffney

36/11 A simple mathematical model for investigating the effect of cluster roots on plant nutrient uptake

Zygalakis

Roose

Neukirch

Frelat

Goriely

Maurini

Style

Cocks

Peppin

Wettlaufer

39/11 An asymptotic theory for the re-equilibration of a micellar surfactant solution
Griffiths

Bain

Breward

Chapman 
43/11 Coupling fluid and solute dynamics within the ocular surface tear film: a modelling study of black Line osmolarity

Zubkov

Breward

Gaffney

44/11 A prototypical model for tensional wrinkling in thin sheets

Davidovitch

Schroll

Vella

Adda-Bedia

Cerda

45/11 A fibrocontractive mechanochemical model of dermal wound clo-

Murphy sure incorporating realistic growth factor

Hall

Maini

$\mathrm{McCue}$

McElwain

46/11 A two-compartment mechanochemical model of the roles of trans-

Murphy forming growth factor $\beta$ and tissue tension in dermal wound healing

Hall

Maini

McCue

McElwain

47/11 Effects of demographic noise on the synchronization of a metapop-

Lai ulation in a fluctuating environment

Newby

Bressloff

48/11 High order weak methods for stochastic differential equations

Abdulle based on modified equations

Cohen

Vilmart

Zygalakis

49/11 The kinetics of ice-lens growth in porous media

Style

Peppin

50/11 Wound healing angiogenesis: the clinical implications of a simple

Flegg mathematical model

Byrne

Flegg

McElwain

Copies of these, and any other OCCAM reports can be obtained from:

Oxford Centre for Collaborative Applied Mathematics Mathematical Institute

24 - 29 St Giles'

Oxford

OX1 3LB

England

www.maths.ox.ac.uk/occam 